\title{
Simulating Flocks on the Wing: The Fuzzy Approach
}

\author{
Iztok LEBAR BAJEC ${ }^{\text {a,*}}{ }^{*}$, Nikolaj ZIMIC ${ }^{a}$ and Miha MRAZ ${ }^{a}$ \\ a University of Ljubljana, \\ Faculty of Computer and Information Science, \\ Trzaska cesta 25, \\ SI-1000 Ljubljana,
}

Slovenia.

\begin{abstract}
Traditionally the systematic study of animal behaviour using simulations requires the construction of a suitable mathematical model. The construction of such models in most cases requires advanced mathematical skills and exact knowledge of the studied animal's behaviour. Exact knowledge is rarely available. Usually it is available in the form of the observer's linguistic explanations and descriptions of the perceived behaviour. Mathematical models thus require a transition from the linguistic description to a mathematical formula that is seldom straightforward. The substantial increase of the processing power of personal computers has had as a result a notable progress in the field of fuzzy logic. In this paper we present a novel approach to the construction of artificial animals (animats) that is based on fuzzy logic. Our leading hypothesis is, that by omitting the transition from linguistic descriptions to mathematical formulas, ethologists would gain a tool for testing the existing or forming new hypotheses about 'why' and 'how' animals behave the way they do.
\end{abstract}

Key words: bird, flock, artificial life, boid, animat, fuzzy logic, fuzzy animat

\section{Introduction}

In nature there exists a phenomenon of aggregation. Most of our surroundings, both inanimate and animate, represent different forms of aggregations.

\footnotetext{
* Corresponding author (Lebar Bajec, I.).

e-mail: iztok.bajec@fri.uni-lj.si,web: http://lrss.fri.uni-lj.si/people/ilbajec/, tel: +386 14768 785, fax: +38614264 647 .
} 
At the most basic level, an aggregation is a collection of parts or units which form some coherent, often cohesive, whole (Parrish et al., 1997). For example, at the most basic level, the landscape in which we live is an aggregation of inanimate objects of all shapes and sizes that was mainly formed by physical sorting. On the other hand, within the animate world, aggregations can result from physical sorting (i.e. passive aggregation) or can form around an attractive source (i.e. active aggregation) (Parrish et al., 1997). In the latter case the aggregation may be formed and maintained by the mutual attraction of members. In such cases, when the source of attraction is the group itself, we define this behaviour as congregation (Turchin, 1997; Parrish et al., 1997). Examples of animal congregations abound, but the most commonly known are flocks of birds, schools of fish and swarms of insects.

Some of the holy grails of animal congregation research are represented by the search for the 'why', the 'how' and the three-dimensional structure of congregations. Although all of the above questions still remain largely unsolved, many features shared by animal congregations, regardless of species or circumstance, are readily known (Parrish et al., 1997):

- congregations have usually very distinct edges,

- many types of animal congregations, particularly when on the move (e.g. flocks, schools, herds) have fairly uniform densities,

- congregations which exist largely as groups of uniform density are often also polarized, with all members facing in the same direction,

- within the volume of the group, polarized or not, individuals have the freedom to move with respect to their neighbours,

- many congregations display coordinated movement patterns of an almost balletic nature.

\subsection{Flocks on the wing}

Most of the animal congregation research is highly dependent on collecting (Jaffe, 1997; Heppner, 1997) large sets of four-dimensional data (i.e. three in space and one in time). For reasons of easier and more fruitful tracking of confined objects (Parrish et al., 1997; Heppner, 1997), fish congregations have been a frequent research theme. On the other hand, bird congregations, for obvious reasons of highly difficult and almost luck-dependent data collection (Heppner, 1997), are among the most widely observed, yet least understood phenomena (Heppner, 1974). According to Heppner (1997), this may be one of the reasons why there is a current of imaginative speculation, and lively controversy in literature on bird congregation structure and internal dynamics.

To help conquer this, Heppner (1974), in his pioneering work, defined the terms 


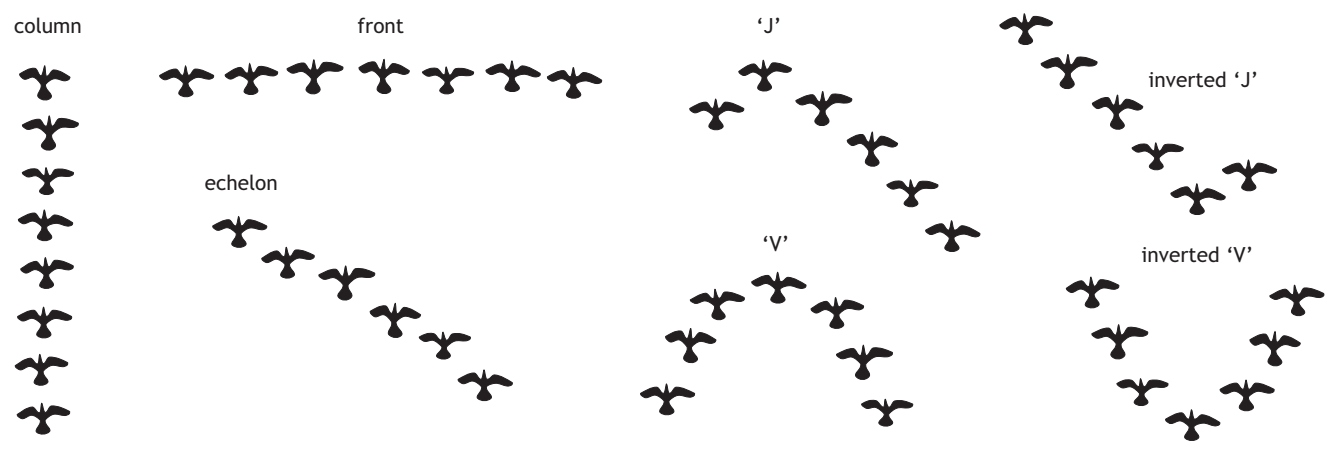

Fig. 1. Line formations: column, front, echelon, ' $\mathrm{J}$ ', 'V', inverted ' $\mathrm{J}$ ' and inverted 'V' (Heppner, 1974).
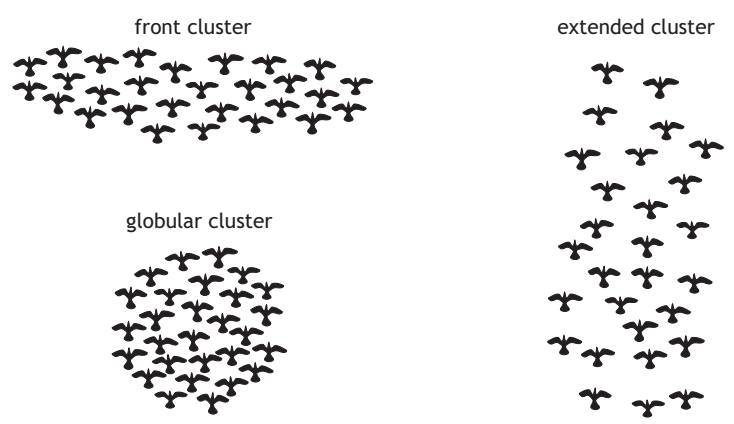

Fig. 2. Cluster formations: front cluster, globular cluster, extended cluster (Heppner, 1974).

flight aggregation ${ }^{1}$ and flight flock ${ }^{2}$, and used them to devise a taxonomy of flight formations. According to his study there are two major classes of flight formations: line formations and cluster formations.

Line formations (Fig. 1) are groups of relatively large birds, such as waterfowl and pelicans, flying in a single line, or joined single lines. Typically they are approximately two-dimensional and show a rather high degree of regularity in spacing and alignment. In line formations birds fly in a single line, one behind the other (column), one beside the other (front) or staggered stepwise from the bird at the head of the formation (echelon). In line formations birds also fly in joined single lines; left and right echelons joined at the tip of the formation (' $J$ ' and ' $V$ ') or at the tail of the formation (inverted ' $J$ ' and inverted ' $V$ '). In $\mathrm{a}^{\prime} \mathrm{V}$ ' and inverted ' $\mathrm{V}$ ' formation the left and right echelon are approximately the same size, whereas in a ' $\mathrm{J}$ ' and inverted ' $\mathrm{J}$ ' formation one is considerably

$\overline{1}$ A group of flying birds, lacking coordination in turning, spacing, velocity, flight direction of individual birds and time of take-off or landing, assembled in a given area. For reasons of consistency hereafter a bird aggregation.

2 A group of flying birds, coordinated in one or more of the following parameters of flight: turning, spacing, velocity, and flight direction of individual birds, and time of take-off and landing. Hereafter bird congregation and flock will be used interchangeably. 
larger.

Cluster formations (Fig. 2) are relatively large flocks of small birds, like sandpipers, characterized by development in the third dimension, and rapid, apparently synchronous turns. In cluster formations birds are typically distributed over a three-dimensional space of an irregular spheroidal shape that is: as wide as it is long (globular cluster), wider than it is longer (front cluster), or longer than it is wider (extended cluster). Birds flying in globular clusters generally fly in apparent close order and can be seen making very rapid turns. Similarly, front clusters tend to have very precise spacing and turning. However, birds flying in extended clusters tend to be rather disorganized, with frequent breakoffs and shifts of position.

While observing line formations, one is impressed by the precision with which relatively small numbers of large birds maintain themselves in accurate spatial alignment and angular orientation with their flockmates. On the other hand, while observing cluster formations, the attention is drawn to the coordination that enables large numbers of small birds, flying in close order, to wheel and turn without suffering mid-air collisions. As a result, bird congregation research is mostly driven by the questions 'why' and 'how' (Heppner, 1997). The former is usually expressed in association with line formations, whereas the latter in conjunction with cluster formations. In the first case the primary interest is the functional significance of formation flight. In the second the attention is given to the synchrony, or apparent synchrony, in the turning movements and the necessity or presence of a leader guiding these manoeuvres. However, the formidable technical challenges at data collection pushed researchers into thinking of new ways to explore the dynamic beauty of bird congregations - new ways of studying their structure and dynamics, and new ways to test hypotheses about 'why' and 'how' birds congregate.

\subsection{Simulating flocks}

In the mid 1980s, different papers appeared, suggesting that coordination in flocks might be achieved by the application of the mathematics of nonlinear dynamics (Okubo, 1986) and that flocking might be an emergent property arising from individuals following simple rules of movement (Heppner, 1987). At the same time, but working in another field of study, namely computer graphics, Reynolds (1987) published a ground-breaking seminal paper that first presented a computer flight flock simulation. In his study a collection of individuals whose behaviour is governed by three simple rules based on geometrical calculations, demonstrates flocking behaviour that is typical for flying birds. Without knowing about Reynolds's work, Heppner joined forces with a mathematician Grenander and published a paper (Heppner and Grenander, 
1990) presenting a computer flight flock simulation. In their work the flock was a self-organizing collection of individuals, whose behaviour was based on stochastic nonlinear differential equations. Both simulations base their assumptions on common grounds and model the behaviour of individuals on the, at times contradictory, clues of attraction and repulsion.

With the exception of some work on evolution of flocking behaviour (Reynolds, 1993a,b, 1994; Spector and Klein, 2002; Spector et al., 2003), and Heppner's unpublished work on flock take-off and landing (Heppner, 1997), papers regarding computer flock simulations subsided after 1990. We believe that the primary reason lies hidden in the mathematical nature of these simulations as well as in the amount of work required to master the effects that parameter changes have on the displayed behaviour. Even Heppner and Grenander (1990) admit that the interesting patterns were discovered serendipitously and that considerable trial-and-error experimentation was needed before flock-like behaviour was produced. Beside this, one can hardly imagine that congregating birds flying at speeds of $150 \mathrm{~km} / \mathrm{h}$ (Heppner, 1997) have the time or the ability to perform sophisticated or time-intensive mathematical calculations. Even Parrish et al. (1997) state that there must be simple traffic rules for species' engaging in collective movement. To continue, we can hardly imagine that birds are able to perceive precise (i.e. crisp) information (e.g. distance) on which all of the existing mathematical models are based. Furthermore, the animal congregation research audience is predominantly composed by ethologists, not mathematicians. This means that the mathematical nature of the existing models makes them difficult to understand by the audience they were designed for. Even if one makes them as black-box modules and allows ethologists only to change the values of parameters, this would not suffice for truthtesting. Truth-testing any sort of simulation that purports to represent natural behaviour is extremely difficult, and has not often been done, especially in behaviour. Models are usually too crude, or have too many special conditions to be readily tested with real-world data. We believe this is why ethologists have difficulties in using the models for testing the existing hypotheses or forming new ones.

We feel that flock-like behaviour could be much more easily described by using simple linguistic descriptions (e.g. collections of if-then rules). In fact, the existing knowledge about the behaviour of flocks is usually available in the form of the observer's linguistic descriptions and explanations of the perceived behaviour ${ }^{3}$. The existing models were arrived at by approximating such linguistic descriptions using mathematical models. Moreover, considerable amounts

3 "It seems likely that if a bird, say, to the left and in front of another bird turned suddenly in front of the trailing bird, the trailing bird would have time to react, and turn in the same direction, avoiding collision." From personal correspondence with Frank Heppner. 
of advanced mathematical skills were required for the transition from the linguistic descriptions to a mathematical model. Fuzzy logic (Zadeh, 1965) is a very popular, successful and widely spread approach for modelling processes, for example fire spread prediction (Mraz et al., 1999; Vakalis et al., 2004a,b), which are too complex for classical mathematical methods. We feel that by using fuzzy logic to describe the simulated animal's behaviour we could make the transition from the linguistic description to the actual behaviour much shorter and much more understandable. This way the animal congregation research audience would gain a tool that enabled them to construct simulated animals through which they could form hypotheses and test their theories about 'why' and 'how' animals congregate.

In the following paper we will, by using the study case of flight flocks, present the current state of the fuzzy animat construction framework. This framework will eventually allow ethologists to construct artificial counterparts of the animals they are interested in by using simple linguistic descriptions. In section two we will present the simulated bird, the universe it lives in, the way it perceives it, its drives, and show the way it behaves. In section three we will show that by using fuzzy logic and common sense about the behaviour of flocking birds we were able to achieve a comparable and in certain cases more natural behaviour than by using the traditional mathematical models. We will conclude the paper with a short discussion about the usability of such a tool and present our future work.

\section{Methods}

Simulating animal behaviour is a complex task, requiring thorough knowledge about the behaviour of the modelled animal. However, the behavioural repertoire displayed by an animal is typically so large that even ethologists are at times unable to form hypotheses about the actions guiding the displayed behaviour, not to mention the reasons that initiated it. Exact knowledge is thus usually not available, and in cases when knowledge is available, it usually cannot be truth-tested. The linguistic descriptions and explanations of the perceived behaviour generally bear a fair amount of influence by the observer and are consequently uncertain or ambiguous per se. Knowledge about an animal's behaviour can as a consequence be best described as uncertain, ambiguous or fuzzy. This is where fuzzy logic, with its ability to model using uncertain knowledge and process uncertain data, shows its potential. 


\subsection{The artificial bird}

Regardless of the method used when modelling an animal, we first need to abstract its basic characteristics. Most of us accept or infer that every animal exists in time and space, and is surrounded by inanimate and animate objects (i.e. the universe). We also presume that animals have senses (i.e. sight, hearing, smell, etc.) through which they have the ability to perceive the current state of the universe. An animal is, through actions (e.g. movement), capable of influencing its internal state and the state of the universe. Regarding the animal's current internal state (e.g. fear) only certain data from the universe (e.g. predator location) are important and the animal's drive is to optimize (e.g. minimize) the rate of their occurrence. The animal selects actions (e.g. fleeing) that satisfy its drives. Regarding its current internal state and the most pressing drives the animal performs a sequence of movements that will accomplish a combination of these actions (action selection). A model that takes into account the above characteristics is commonly referred to as artificial animal or animat (Wilson, 1985).

According to the above discussion, our artificial bird's state at a point in time is given by its current position in space ${ }^{4}$, flight direction, flight speed and internal state. The artificial bird's next state is derived through a three-stage transition function that simulates perception of the universe, drives and action selection (see Lebar Bajec (2002); Lebar Bajec et al. (2003a,b); Mraz et al. (2004) for a detailed discussion).

\subsection{The universe}

Reynolds (1987) in his study modelled the universe as a collection of animats ${ }^{5}$ and obstacles representing inanimate objects. He states that obstacles and the animats' attempts to navigate around them increase the apparent complexity of the displayed behaviour. In his paper he suggests that the complexity of real flocks might be due largely to the complexity of the natural environment (Reynolds, 1987). More recently ${ }^{6}$, however, he has acknowledged that the lifelike, unpredictable behaviour of animats emerges from the complex adaptive nature of the model. Heppner and Grenander (1990), on the other hand, mod-

\footnotetext{
4 Although it is relatively easy to extend our model to three-dimensions we, for reasons of simpler explanation and presentation, in this paper assume that the space is a two-dimensional plane.

5 Reynolds refers to his simulated bird like, "bird-oid" objects generically as boids, but in this paper we shall refer to them simply as animats. The proof that a boid is a specialized animat can be found in (Lebar Bajec, 2002).

6 http://www.red3d.com/cwr/boids/applet/
} 
elled the universe as a collection of animats and excluded inanimate objects, but added a special influence which is intended to simulate the effects of wind gusts and random local disturbances. They state that the latter was of crucial importance for flock-like behaviour (Heppner and Grenander, 1990).

Since we are interested in producing a self-organizing flight flock, we model the universe as a collection of animats. At this stage of our fuzzy animat construction framework we also omit obstacles. We justify their omission with the fact that flocks form and exist also in open spaces, where there are no obstacles. For the time being we are inclined not to simulate the effects of wind gusts and random local disturbances, but it would be interesting to test the effect of their inclusion. Similarly to Reynolds (1987) and Heppner and Grenander (1990), we assume a constant universe - even though every animat's state changes through time the number of simulated animats stays constant.

\subsubsection{Modelling perception}

Heppner and Grenander (1990) give each animat complete and precise information about the universe. In doing so they make an unrealistic assumption because real birds have limited and imperfect perceptive capabilities (e.g. nearby flockmates hide those far away, thus the bird can not visually perceive them). Reynolds (1987), admitting that a bird's perception of the world is severely limited by occlusion, models perception using a volume, within which the animat has the ability to sense flockmates. But the sensed information is precise - giving the animat precise information about the state of the flockmates (i.e. their exact distance, position, flight speed and flight direction). Even though Reynolds (1987) states that his perception model tries to make available approximately the same information that is available to a bird as the end result of perceptual and cognitive processes, we believe that his approach, although better, is still unrealistic. We believe that a bird's visual perception is not limited only by occlusion, but also by the fact that the ability to sense distance, apart from being affected by the degree of binocular overlap, decreases with distance itself.

Even though it is likely that as hydrodynamic perception is important in fish, aerodynamic perception is important in birds, we currently model only visual perception. Although it is still an unrealistic model, we adopt a slightly modified Reynolds's (1999) approach of localized visual perception. The main difference is in the returned information. We model visual perception as a visual volume defined by the visual range $V_{\mathrm{R}}$ and per eye visual field $V_{\text {FOV }}$ of the animat (see Fig. 3). At the time being we are using the visual range of seven body lengths and the per eye visual field of $150^{\circ}$ with no binocular overlap. The animat thus has a blind area of $60^{\circ}$ behind it, which corresponds 


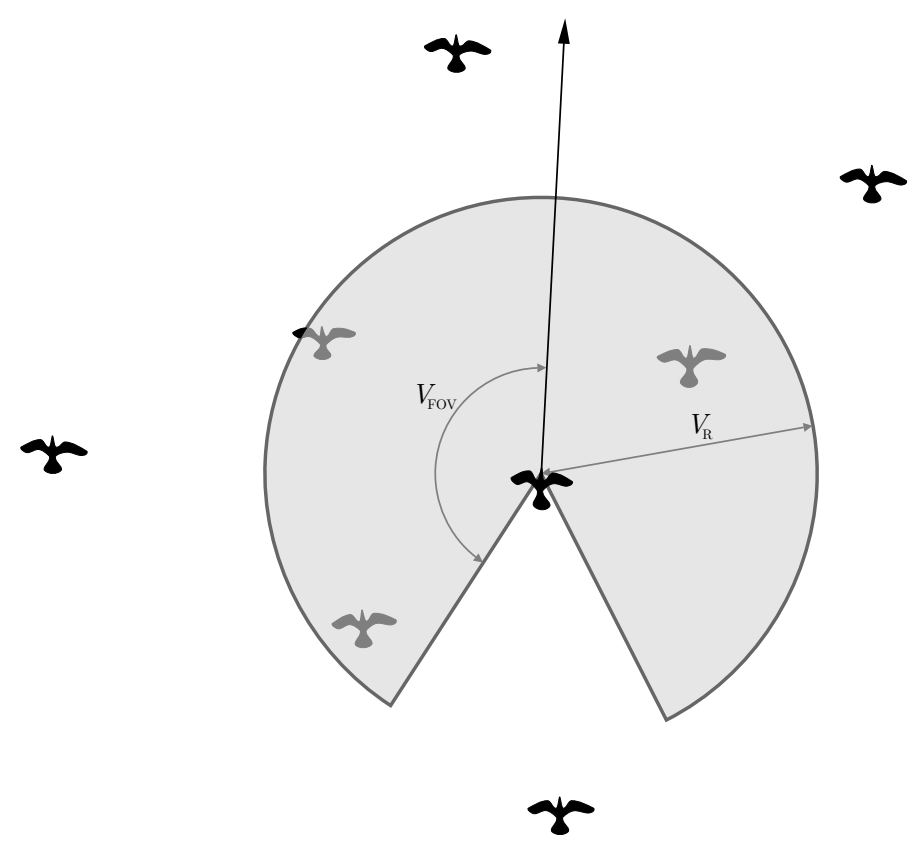

Fig. 3. Localized perception. The black arrow represents the flight direction. The shaded area represents the visual volume defined by the visual range $V_{\mathrm{R}}$ and per eye visual field $V_{\mathrm{FOV}}$. The perceived flockmates are depicted in a dark grey colour.

closely to the values reported by Heppner et al. (1985). Instead of giving full information about the perceived flockmates we give only their distance, angular offset, relative difference in flight direction and relative difference in flight speed. We justify this by the fact that using visual perception, a bird can sense only distance and angular offset of a flockmate, but through cognitive processes and tracking it can judge the relative difference in flight speed (i.e. if the flockmate is moving faster, slower or with the same speed) and their relative difference in flight direction (i.e. if the flockmate is flying more to the left, more to the right or in the same direction ${ }^{7}$. We reserve the modelling of the binocular overlap and imperfect visual perception for our future work.

\subsection{Modelling drives}

Even though in his latest studies (Reynolds, 1999, 2001) presented drives ${ }^{8}$ through the combination of which we can achieve complex behaviours, he states that flight flock behaviour can be achieved using only three drives,

7 It could be argued that by using this information one can calculate the same precise information as in Reynolds's (1999) case, but we strongly object to any mentioning that a bird has precise or full information about its global position in space, absolute flight speed or absolute flight direction.

${ }^{8}$ In his paper he uses the name steering behaviours, but for reasons of consistency and clarity we shall use the term drives. 
namely separation, cohesion and alignment. Cohesion simulates attraction toward flockmates and is modelled as the animat's tendency to fly towards the centroid of the perceived flockmates. Separation simulates repulsion away from flockmates and is modelled as the animat's tendency to fly away from the perceived flockmates. These two drives represent the so-called attractionrepulsion scheme. Alignment, on the other hand, tries to produce polarization and is modelled as the animat's tendency to change its flight direction and flight speed, so that it corresponds to the average flight direction and flight speed of its perceived flockmates. We agree with the three drives, but find Reynolds's (1987) use of the centroid of the perceived neighbours somewhat questionable. The centroid of the perceived neighbours is a mathematical construct and it is difficult to believe that a bird has knowledge of such constructs or uses them to compute its action. It was Pliny (Rackham, 1933; Heppner, 1997) who noted that "it is a peculiarity of the starling kind that they fly in flocks and wheel round in a sort of circular ball, all making towards the centre of the flock". However, in our opinion, birds might not have any idea about the centre of the flock and their making towards it might be just an emergent property by itself.

Heppner and Grenander (1990) also modelled three, but different, drives, namely homing, velocity regulation and interaction. Homing simulates the attraction of the roosting point and is modelled as the animat's tendency to fly toward the roosting point. This tendency drops to zero if the animat is close enough (i.e. a predefined distance) or too far away (i.e. a predefined distance) from the roosting point (see Heppner and Grenander, 1990, Fig. 1). Velocity regulation is modelled as the animat's tendency to fly at a certain predefined preferred speed (see Heppner and Grenander, 1990, Fig. 2). Interaction, on the other hand, combines the attraction-repulsion scheme in one single drive and simulates the actual interaction between animats. If two animats are too close (i.e. a predefined distance) they are repelled, if they are too far (i.e. a predefined distance) they do not influence each other and if they are anywhere in between, they are attracted (see Heppner and Grenander, 1990, Fig. 3).

Attraction towards and repulsion from the flockmates feel natural after all, their mutual coexistence and importance for the congregation's structure has already been suggested by Okubo (1980). On the other hand, velocity regulation used by Heppner and Grenander (1990) feels somewhat strange. This drive is derived from aerodynamic theory; with a given power output and configuration, an aircraft will maintain a constant speed, it will return to that speed if perturbed. A real bird would not have to make decision about this. In our opinion this drive has a questionable effect. Take, for example, an animat that slowed down because it was too close to one of its flockmates. It will speed up. But not because it is trying to catch up with its flock, but because it is returning to its predefined preferred speed. In our opinion this behaviour resembles more to an aggregation of birds that happen to be flying 
together than to a flock of birds that are trying to fly together. Furthermore, it is hardly believable that a bird has a predefined preferred flight speed. Even if it does we hardly believe it is constant in time. We do believe that birds tend to change their flight speed regardless of the speed of the flock, but this might be primarily caused by fatigue or other distractions (e.g. wind gusts). If one wanted to simulate this it would be much better to model fatigue as well (i.e. extend the animat's internal state and take into account energy consumption). Another important feature of groups of uniform density is polarization. Heppner and Grenander (1990) did not model it specifically, but they mention that in certain cases organized flocks maintaining straight direction of flight emerged. We believe that this might be caused by the perception model they used (i.e. all animats have complete and perfect information about the universe). Reynolds (1987), however, tries to model polarization through the drive of alignment.

According to the above discussion we model the three primary drives, the attraction to flockmates, the repulsion from flockmates and polarization with flockmates. In the following subsections we will discuss them in greater detail. When modelling attraction to flockmates we do not use a predefined preferred position like the centroid (Reynolds, 1987). Similarly, when modelling polarization with flockmates we do not use a predefined preferred flight speed (Heppner and Grenander, 1990). Instead we let these properties emerge on their own.

As discussed in subsection 2.2.1, our animat perceives the state of the universe through visual perception only. At any point in time the animat perceives only information about a localized subset of the universe. Since we model the universe as a collection of animats, the animat thus perceives information about its nearby flockmates. The perceived information includes only distance, angular offset, relative difference in flight direction and relative difference in flight speed of the nearby flockmates. At the time being the perceived information is precise and we reserve modelling imperfect perception for our future work. Furthermore, we shall suppose that the animat can act only by changing its flight speed and/or flight direction.

\subsubsection{Attraction to flockmates}

The primary motive of the attraction drive is to stay close to nearby neighbours. Now, imagine a bird that perceives only one neighbour. How would you, in the simplest way possible, describe the action that will keep it close to the perceived neighbour? Assuming that the bird can act only by changing its flight speed and/or flight direction and using common sense most of us would probably state the following: 
(1) in general do not change flight speed or flight direction;

(2) when the perceived neighbour is 'close enough', change neither flight speed nor flight direction;

(3) when the perceived neighbour is 'too far' and 'in front', speed up;

(4) when the perceived neighbour is 'too far' and anywhere to the 'left or behind', turn toward it and slow down;

(5) when the perceived neighbour is 'too far' and anywhere to the 'right or behind', turn toward it and slow down.

If we look carefully at our description we notice that the resulting action depends only on the perceived neighbour's distance (i.e. 'close enough', 'too far') and position (i.e. 'in front', 'left or behind', 'right or behind'). But what do 'close enough', 'too far', 'in front', etc. mean? Does 'in front' perhaps address the precise moment when the perceived neighbour is positioned at an angular offset of $0^{\circ}$ ? What about $5^{\circ}$, is the perceived neighbour then not 'in front'? As we can see 'in front' is an imprecise property and constructing a mathematical model from a description that builds on such imprecise properties is a challenging task, which usually requires advanced mathematical skills. Then again, because 'close enough', 'too far', 'in front', etc. are imprecise properties and do not represent crisp values like $0^{\circ}$ or $5^{\circ}$ we can label them as vague or fuzzy values. Thanks to Zadeh (1965), who introduced fuzzy sets (see Appendix A), we can define such values ${ }^{9}$. Constructing a model now becomes simple. All that we have to do is rewrite the description as a collection of easily understandable if-then rules and the necessary action can be computed by applying fuzzy logic (see Appendix B).

Consider, for example, the fuzzy value 'close enough'. As already said, we can represent it with a fuzzy set. In other words, this means that a fuzzy value is uniquely defined by its membership function (see Appendix A). In our case, the latter provides the degree to which a real number satisfies the property 'close enough' (i.e. its degree of membership). But because the interpretation of 'close enough' is subjective, there is no unique membership function; it is left to the modeller to decide what it should be like. Thus the question is: what did we have in mind with 'close enough'? Let us assume that we consider the perceived neighbour as 'close enough' if its distance is $40 \%$ of the visual range or less. As the distance increases we consider the perceived neighbour less and less 'close enough' and eventually, when it gets out of the visual range, we do not consider it as 'close enough' at all. This translates in the membership function that is presented on Fig. 4a. However, this is only one of the many possible interpretations of 'close enough' and an interesting question is: when does a bird consider its neighbour to be 'close enough'? Here comes into play the expertise of field ornithologists, which, with a tool such as this, can easily

$\overline{9}$ In fact, according to the latest fuzzy sets related literature (Lee et al., 2004) a fuzzy value is a fuzzy set defined in the real number domain. 

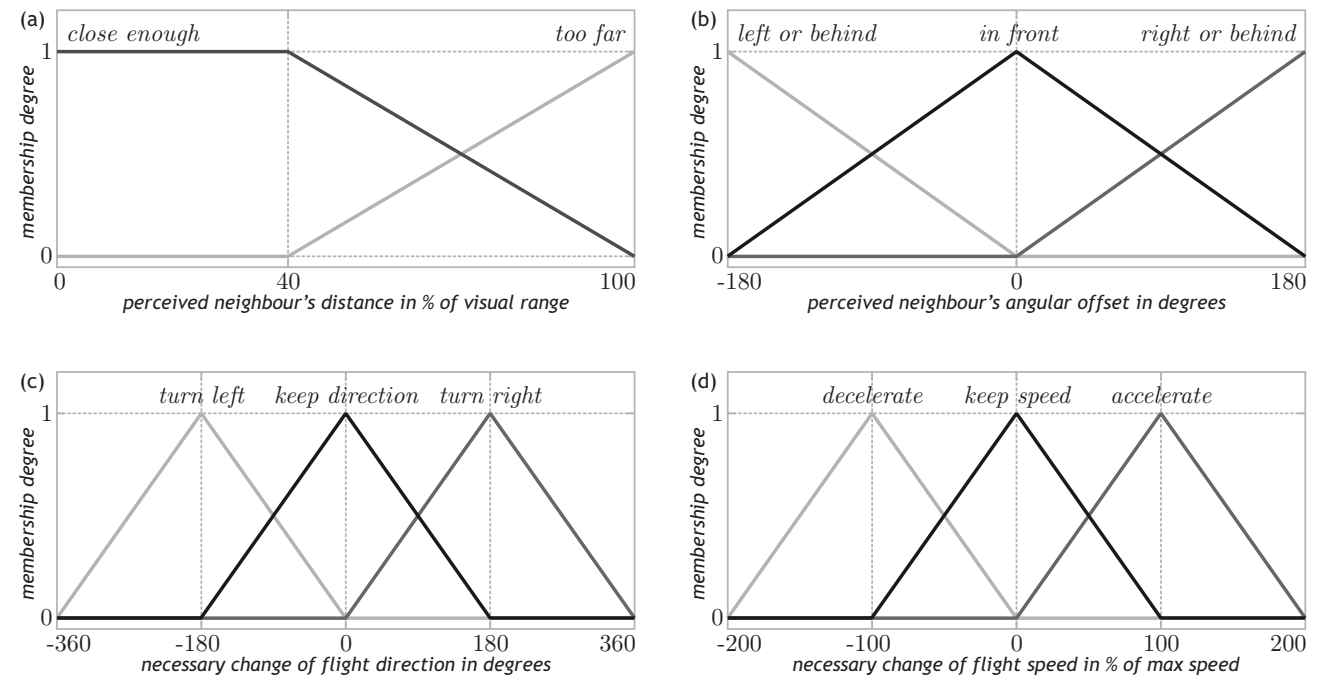

Fig. 4. Membership functions of the fuzzy values 'close enough', 'too far' (a), 'in front', 'left or behind', 'right or behind' (b) 'turn left', 'keep direction', 'turn right' (c) 'decelerate', 'keep speed', 'accelerate' (d) for the case of the attraction drive.

translate their observational knowledge into simulation models.

In a similar fashion we define the fuzzy values 'too far', 'in front', 'left or behind' and 'right or behind' (Fig. 4a,b). If we introduce the fuzzy values 'keep direction', 'turn left', 'turn right', 'keep speed', 'accelerate' and 'decelerate' (Fig. 4c,d) to represent the actions of keeping the same flight direction, performing a left turn, etc. our description can be rewritten in the form of the following set of if-then rules:

a1: if (distance is close enough) then

(flight direction is keep direction),

a2: if (distance is too far) then

(flight direction is keep direction),

a3: if (distance is close enough) then

(flight speed is keep speed),

a4: if (distance is too far) then

(flight speed is keep speed),

a5: if (distance is too far) and (position is in front) then

(flight speed is accelerate),

a6: if (distance is too far) and (position is left or behind) then

(flight direction is turn left),

a7: if (distance is too far) and (position is left or behind) then

(flight speed is decelerate),

a8: if (distance is too far) and (position is right or behind) then

(flight direction is turn right), 
a9: if (distance is too far) and (position is right or behind) then

(flight speed is decelerate).

The first four rules (a1-a4) model the assumption that a bird in general tends not to change its flight direction or flight speed; item (1) in our description. Rules a2 and a4 model the assumption that a bird, in order to keep close to a neighbour that is already close enough, does not need to do anything; item (2). Rule a5 models the assumption that a bird, in order to catch up with a neighbour that is in front of it but too far, needs only to speed up; item (3). The last four rules (a6-a9) model the assumption that a bird, in order to get close to a neighbour that is too far but positioned sideways or behind, needs to turn toward it and slow down; items (4) and (5).

Together the rules can be used to model the animat's attraction drive. When the animat perceives only one neighbour, its action is computed by applying fuzzy logic (see Appendix B) on each of the rules and combining the rule outputs. The resulting action will keep the animat close to the perceived neighbour. When the animat perceives more than one neighbour the rules are evaluated for each neighbour independently (i.e. as if the animat perceived only that neighbour) and all outputs are combined (see Appendix C). The resulting action is a combination that will satisfy the animat's drive to keep close to all of the perceived neighbours. In any case the resulting action is the required change in flight direction (given as the angle of turning) and the required change in flight speed (given as the \% of maximal speed).

\subsubsection{Repulsion from flockmates}

The primary motive of the repulsion drive is to stay away from collisions. We shall again assume that our hypothetical bird perceives only one neighbour, except that this time we are interested in the action that will keep it away from colliding with that neighbour. Using common sense most of us would describe the bird's behaviour in the following way:

(1) in general do not change flight speed or flight direction;

(2) when the perceived neighbour is 'far enough', change neither flight speed nor flight direction;

(3) when the perceived neighbour is 'too close' and anywhere 'behind', speed up;

(4) when the perceived neighbour is 'too close' and 'in front or right', turn away from it and slow down;

(5) when the perceived neighbour is 'too close' and 'in front or left', turn away from it and slow down.

Once more we can notice that in our description the resulting action depends only on the perceived neighbour's distance and position. Therefore, as we did 

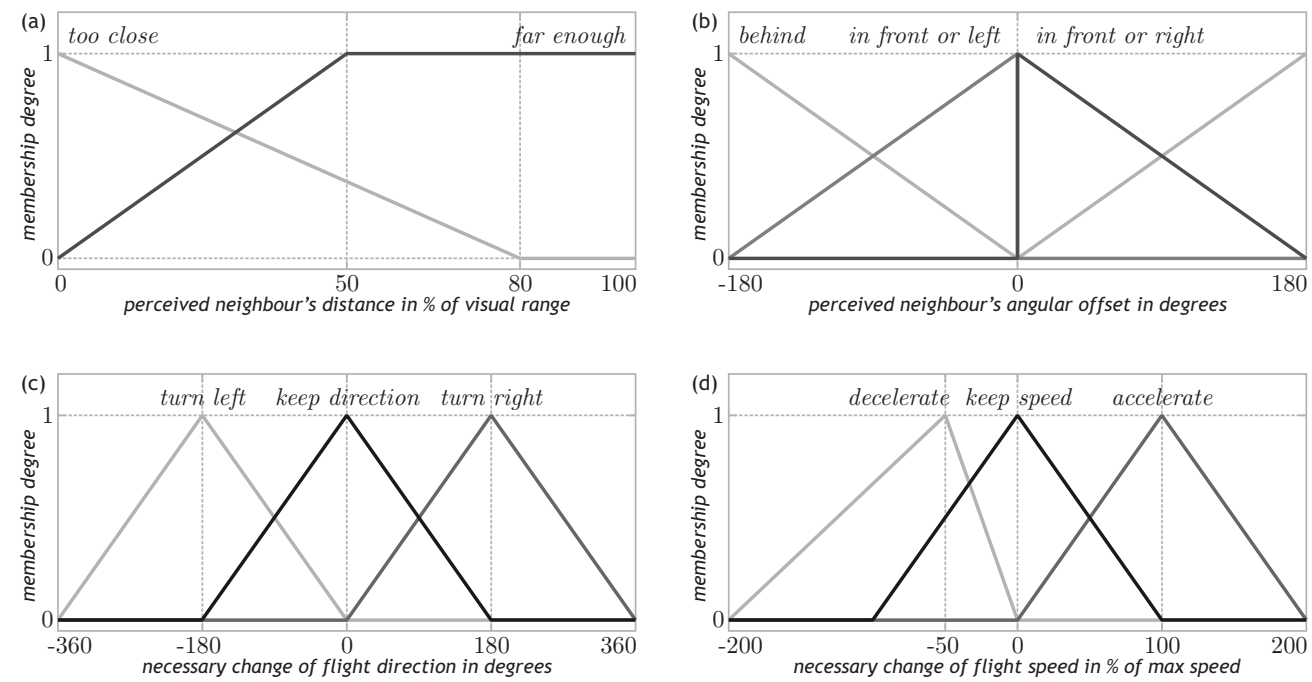

Fig. 5. Membership functions of the fuzzy values 'too close', 'far enough' (a), 'behind', 'in front or left', 'in front or right' (b) 'turn left', 'keep direction', 'turn right' (c) 'decelerate', 'keep speed', 'accelerate' (d) for the case of the repulsion drive.

for the case of the attraction drive (subsection 2.3.1), we first define the fuzzy values 'far enough', 'too close', 'behind', 'in front or right' and 'in front or left' (Fig. 5a,b). Then we introduce the fuzzy values that represent the actions of keeping the same flight direction, performing a left turn, etc. (Fig. 5c,d). After completing these two steps our description can be rewritten in the form of the following set of if-then rules:

r1: if (distance is far enough) then

(flight direction is keep direction),

r2: if (distance is too close) then

(flight direction is keep direction),

r3: if (distance is far enough) then

(flight speed is keep speed),

r4: if (distance is too close) then

(flight speed is keep speed),

r5: if (distance is too close) and (position is behind) then

(flight speed is accelerate),

r6: if (distance is too close) and (position is in front or left) then (flight direction is turn right),

r7: if (distance is too close) and (position is in front or left) then (flight speed is decelerate),

r8: if (distance is too close) and (position is in front or right) then (flight direction is turn left),

r9: if (distance is too close) and (position is in front or right) then (flight speed is decelerate). 
For each of the perceived neighbours our animat applies fuzzy logic to the above set of rules and works out the action that should be taken to keep away from colliding with that neighbour. These actions are then combined into a single resulting action (see Appendix $\mathrm{C}$ ), which satisfies the animat's drive to stay away from colliding with any of the perceived neighbours. The resulting action gives the required changes in flight direction and flight speed.

\subsubsection{Polarization with flockmates}

The polarization drive's motive is to keep approximately the same flight speed and flight direction as the perceived flockmates. If we yet again assume that the hypothetical bird perceives only one neighbour then we can describe the actions that will keep the bird flying in approximately the same direction and with approximately the same speed as the neighbour:

(1) in general do not change flight speed or flight direction,

(2) when the perceived neighbour is 'too far' or 'too close', change neither flight speed nor flight direction;

(3) when the perceived neighbour is at a 'good' distance and flying in the 'same direction', keep flight direction;

(4) when the perceived neighbour is at a 'good' distance but flying more to the 'left', turn left;

(5) when the perceived neighbour is at a 'good' distance but flying more to the 'right', turn right;

(6) when the perceived neighbour is at a 'good' distance and flying with the 'same speed', keep flight speed;

(7) when the perceived neighbour is at a 'good' distance but flying 'slower', slow down;

(8) when the perceived neighbour is at a 'good' distance but flying 'faster', speed up.

As a contrast to the attraction and repulsion drives (sections 2.3.1 and 2.3.2) we notice that in this case the action depends on the perceived neighbour's distance, difference in flight direction and difference in flight speed. By defining and introducing the required fuzzy values (Fig. 6) we can rewrite the above description in the form of a set of if-then rules:

p1: if (distance is too far) then

(flight direction is keep direction),

p2: if (distance is too close) then

(flight direction is keep direction),

p3: if (distance is too far) then

(flight speed is keep speed), 

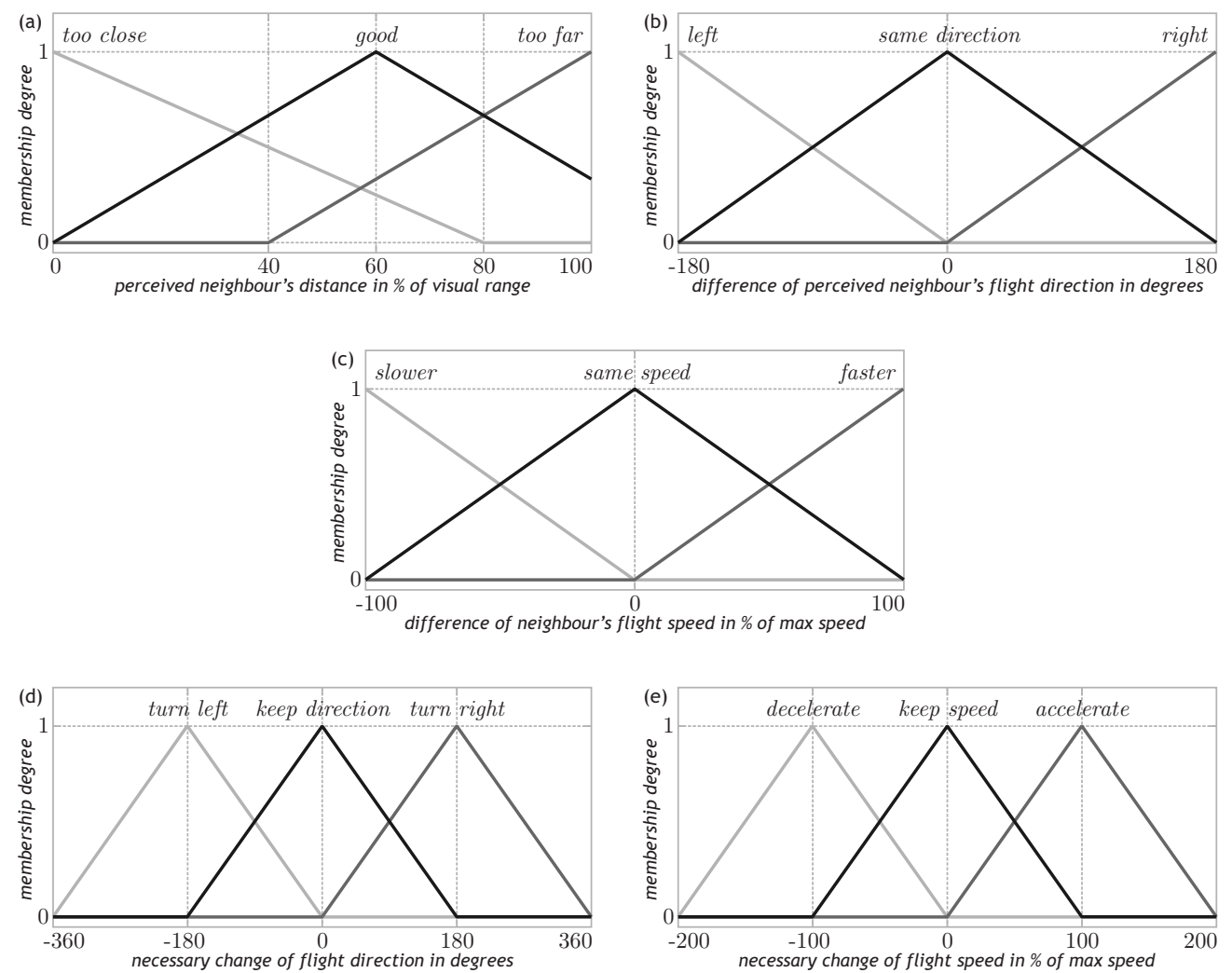

Fig. 6. Membership functions of the fuzzy values 'too close', 'good', 'too far' (a), 'left', 'same direction', 'right' (b) 'slower', 'same speed', 'faster' (c) 'turn left', 'keep direction', 'turn right' (c) 'decelerate', 'keep speed', 'accelerate' (d) for the case of the polarization drive.

p4: if (distance is too close) then

(flight speed is keep speed),

p5: if (distance is good) and (direction is same direction) then

(flight direction is keep direction),

p6: if (distance is good) and (direction is left) then

(flight direction is turn left),

p7: if (distance is good) and (direction is right) then

(flight direction is turn right),

p8: if (distance is good) and (speed is same speed) then

(flight speed is keep speed),

p9: if (distance is good) and (speed is slower) then

(flight speed is decelerate),

p10: if (distance is good) and (speed is faster) then

(flight speed is accelerate), 
Using fuzzy logic on the above set of rules, our animat works out the action that should be taken in order to keep approximately the same flight speed and flight direction as the perceived neighbours (see Appendix C). As for the attraction and repulsion drives the resulting action gives the required changes in flight direction and flight speed.

\subsection{Modelling action selection}

The action selection process simulates the animal's neurological process of selecting the sequence of muscular movements that will accomplish the actions that result from its drives. This process combines, prioritizes, and arbitrates between potentially conflicting actions. Since in our study we do not model the musculoskeletal structure of the bird, we model the action selection mechanism as a combination of the resulting actions. Thus, for each drive a vector is calculated that represents the force needed to accomplish the required action. These forces are then combined by using a weighted sum. The weights are chosen to give the highest priority to the separation drive, followed by the polarization drive, and the lowest priority to the attraction drive. The resulting vector is used to calculate the animat's new flight speed and flight direction. This calculation is subjected to a set of constraints modelling conservation of momentum, viscous damping and the animal's finite amount of available energy. The same approach named geometrical flight was used already by Reynolds (1987, 1999). Heppner and Grenander (1990), who represented actions as vectors, used a similar approach and modelled action selection as a simple sum. But, apart from the Poisson process simulating the effects of wind gusts and random local disturbances, they did not apply any additional constraints.

\section{Results and Discussion}

We verified our model by comparing it with the existing mathematical models and by comparing the displayed behaviour with that seen in natural flocks. Fortunately, Reynolds has recently made available the OpenSteer library ${ }^{10}$ that includes an implementation of his model (Reynolds, 1999), but sadly the model used by Heppner and Grenander (1990) is not available on-line ${ }^{11}$ and thus we had to content ourselves by comparing our and Reynolds's model only. We compared the two models through three sets of experiments, with which

\footnotetext{
$\overline{10 \mathrm{http}: / / o p e n s t e e r . s o u r c e f o r g e . n e t}$

${ }^{11}$ Recently, through personal correspondence, Heppner gave us a printout of the source code used by Heppner and Grenander (1990) and thus a comparison with their model is planned as our future work.
} 


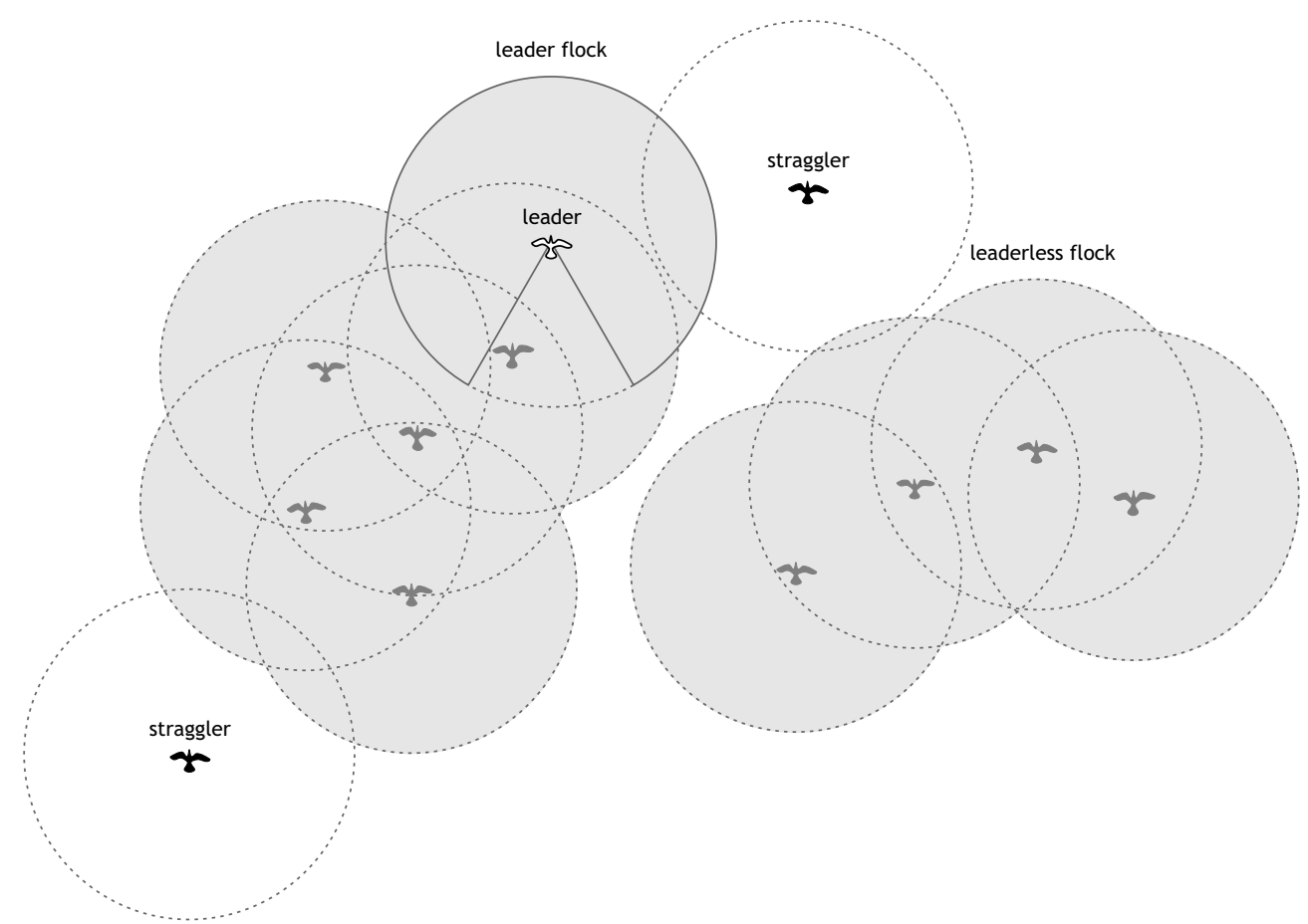

Fig. 7. A leader flock, a leaderless flock and two stragglers. Dashed lines represent the range of potential influence. The shaded area thus encompasses the animats that potentially influence each other and therefore represents the flock extents. The solid line in the leader flock represents the visual volume of the leader and shows that even though it potentially influences other members of the flock it is not influenced by any of them.

we wanted to estimate the flocking ability of the modelled virtual birds and resemblance of their behaviour to that seen in natural flocks.

When estimating the flocking ability we turned to counting the number of animat to animat collisions, the number of flocks, and the number of stragglers. But, wanting to count them algorithmically, we stumbled in the definition of the term flock. According to Heppner (1974), a flight flock is a group of flying birds, coordinated in one or more of the following parameters of flight: turning, spacing, velocity and flight direction of individual birds, and time of take-off and landing. However meaningful and accurate from an ornithologist's point of view this definition is, it gives too little information from an algorithmic point of view. For reasons of computational simplicity and assumptions used when constructing the model, we decided to define a flock in terms of animat proximity. We stated simply that two animats that are close enough to potentially influence each other (i.e. in visual range) are members of the same flock (see Fig. 7). With such an interpretation we can distinguish between flocks and stragglers, where the latter are animats that are not members of any flock. Moreover, by taking into account the perception model used, we can distinguish between leader flocks and leaderless flocks. A leader flock is a flock with at least one leader (i.e. animat that is not influenced by any of its flockmates, 
but influences at least one), whereas a leaderless flock is a flock with no leader (i.e. each animat is influenced by at least one of its flockmates). It should be noted that these definitions represent merely a first-order approximation, but even though they might not be accurate enough from an ornithologist's point of view, they are sufficiently accurate from an algorithmic point of view. It should also be noticed that in no way they suggest or assume the existence of a single and absolute leader, in the sense of a military marching formation. In fact, as Pomeroy and Heppner (1992) reported, when pigeons make a turn, the birds change position such that a bird at the head of a flock will be in the rear of the flock if the flock turns $180^{\circ}$. Therefore the hypothetical absolute leader would be at the side or rear of the flock after a turn, which means that such a model may not be appropriate at all. The distinction between leader flocks and leaderless flocks was introduced only to help algorithmic evaluation of the displayed behaviour. Because most real birds have a very good vision to the rear, with the exception of a blind spot directly to the rear, it is very likely that a bird at a head end of a flock will be influenced by trailers. It is thus sound to assume that in nature mostly leaderless flocks exist and if the animats are to display natural looking behaviour there should be mostly leaderless flocks.

On the other hand, when estimating the resemblance of the emerged flocks to natural flocks we turned to visual inspection of the flight formations (see Fig. 1 and 2) and the presence of the somewhat erratic, unsystematic behaviour seen in natural flocks (Heppner and Grenander, 1990). We also estimated the level of the flock's polarization by measuring the flight speed and flight direction standard deviations and the degree of uniform distribution by measuring the nearest neighbour distance.

In our first set, consisting of eight experiments, 100 animats were initially placed at random locations having random flight directions and flight speeds. In this set of experiments we were primarily interested if our animats were able to self-organize in flocks. To help self-organization (i.e. allow animats to eventually meet other animats) we confined the animats using an invisible boundary (see grey circle in Fig. 9) ${ }^{12}$. Whenever an animat passed this boundary it was forced to turn and eventually return in the confinement without changing its flight speed. In a way this resembles modelling the roosting area used by Heppner and Grenander (1990). Our animats were strikingly good at avoiding collisions and self-organizing in flocks. While in our case in most cases no collisions occurred, Reynolds's model, summed over the whole population of 100 animats, averaged $15.63 \pm 4.5$ collisions in 3000 simulation steps (see Tab. 1). We also noticed, in our case, that, when collisions did occur, they occurred in short periods of time (e.g. 10 collisions in 200 simulation

\footnotetext{
$\overline{12}$ Animated versions of Fig. 9, 12, and 13 are available in MPEG4 format at the first author's web site (http://lrss.fri.uni-lj.si/people/ilbajec/papers/ilb_JTB05.htm).
} 


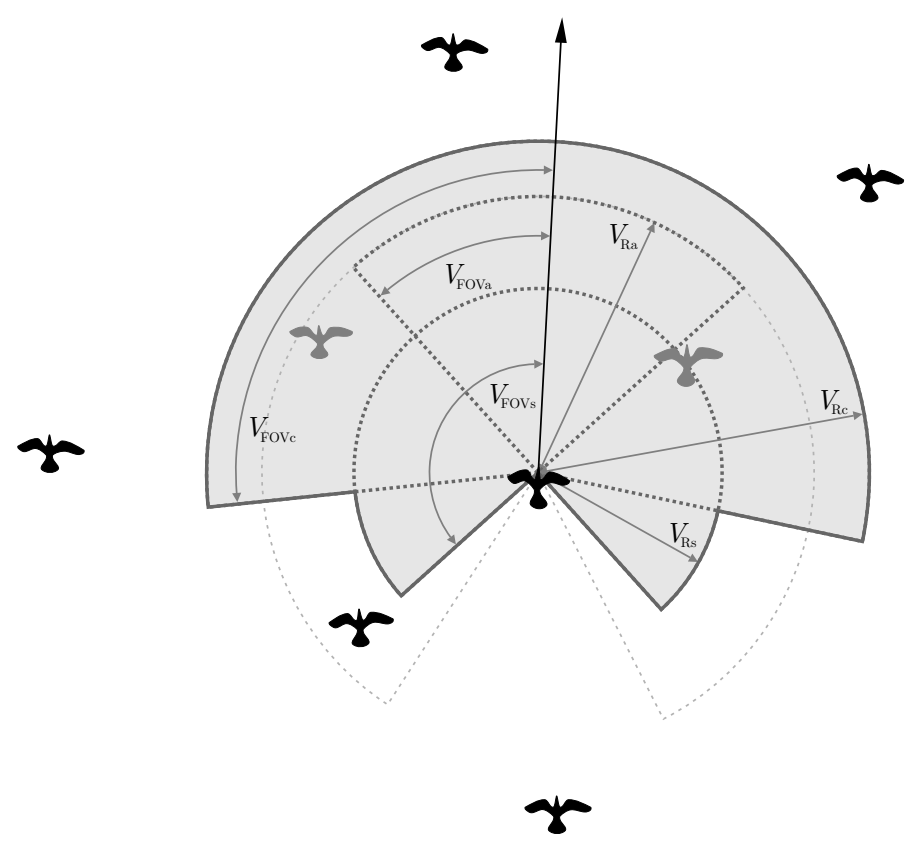

Fig. 8. The perception model that Reynolds used in the OpenSteer implementation of his model (Reynolds, 1999). The black arrow represents the flight direction. The shaded area represents the combined visual volume defined by the distinct separation $\left(V_{\mathrm{Rs}}, V_{\mathrm{FOVs}}\right)$, cohesion $\left(V_{\mathrm{Rc}}, V_{\mathrm{FOV}}\right)$ and alignment $\left(V_{\mathrm{Ra}}, V_{\mathrm{FOVa}}\right)$ drive visual volumes. The perceived flockmates are depicted in a dark grey colour. The light grey dashed line represents the visual volume used in our model.

steps in experiment 03) and were caused by extreme proximity of animats and could be interpreted as side bumping. In Reynolds's case, however, collisions occurred randomly throughout the simulation and were mostly head-on collisions, caused by the merging of flocks. Looking at the simulations in real-time we noticed that in our case both classes of flock formations (i.e. line and cluster formations) emerged, but mostly front cluster ones. Line formations in this set of experiments did not persist for long periods of time. On the other hand, in Reynolds's case the majority of the emerged flocks was of the extended cluster formation whereas line formations did not emerge at all. Figure 10 presents a plot of the number of flocks in experiment 01 for our and Reynolds's model. By comparing the proportion of leaderless flocks it can be noticed that in our case mostly leaderless flocks exist, whereas in Reynolds's case mostly leader flocks exist. This tendency was also noticed in the rest of the experiments (see Tab. 1). The fact that in Reynolds's model mostly leader flocks emerge is primarily caused by the perception model used in OpenSteer (i.e. three distinct visual volumes - one per drive). Not only that the combined visual volume (Fig. 8) gives the animat a two times larger blind area than our model, it also gives the animat a much larger blind area than the values reported by Heppner et al. (1985). As a result it is relatively common that a trailing animat enters the followed animat's blind area and consequently does not influence it any more. The followed animat thus becomes the trailer's leader. If not influenced 


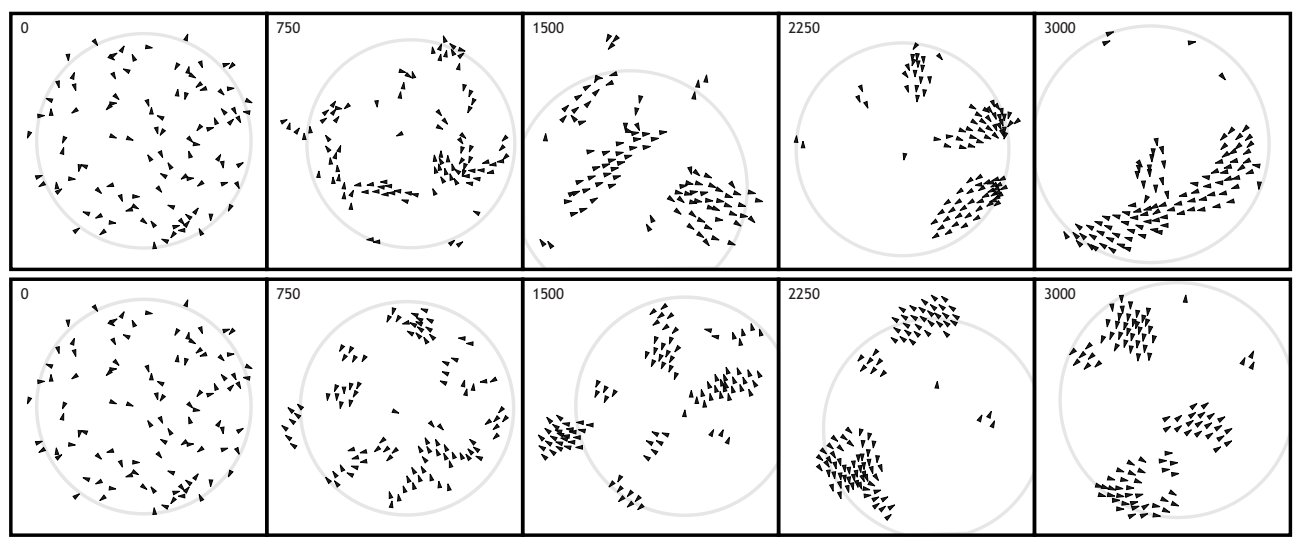

Fig. 9. A comparison of a series of time-equidistant frames from one of the eight experiments for the estimation of flocking ability for Reynolds's (1999) mathematical model (top row) and our fuzzy model (bottom row). The black triangles represent animats, with the apex indicating the flight direction. For reasons of print clarity the triangles were scaled, which means that the apparent overlapping does not necessarily imply a collision. The grey circle surrounding the animats represents the roosting boundary. Whenever an animat crosses this boundary it is forced to turn and eventually return into the roosting area. An animated version of this figure is available at the first author's web site.

by any of its flockmates, the leading animat, because no random local disturbances are modelled, keeps its flight speed and flight direction while all of the flockmates only regulate their flight directions and flight speeds to follow it. Therefore, if not confined, the group will eventually form a regularly distributed and stable extend cluster formation (see frames 667, 1000, and 1333 in Fig. 12 and 1500, 2000 in Fig.13). According to Heppner (1974), the extended cluster formations in nature may simply be bird aggregations in which birds are flying independently toward a common destination. One might be tempted to conclude that this is precisely what we are experiencing here. However, as Heppner states, extended clusters tend to be rather disorganized, with frequent breakoffs and shifts of position, but these features are not to be seen in the leader flocks that emerge from Reynolds's model. This is why we find Reynolds's use of three distinct visual volumes questionable.

In our second set of experiments we were interested if from an initial globular cluster formation, where all animats have the same flight speed and flight direction, natural looking flocks emerge. We used three initial distribution patterns (see Fig. 11), for which we changed the nearest neighbour distance (approximately 2, 4 and 6 body lengths) and initial flight speed (10\%, 50\% and 90\% of maximum flight speed), that is 27 initial states in all. Figure 12 presents a comparison of a series of time-equidistant frames from one of the experiments. In this set of experiments no roosting area was modelled. In Reynolds's case in most of the experiments one stable leader flock of the extended cluster formation emerged, which, in the 10000 simulation steps, stabilized flying at exactly maximum speed with the nearest neighbour distance matching the 

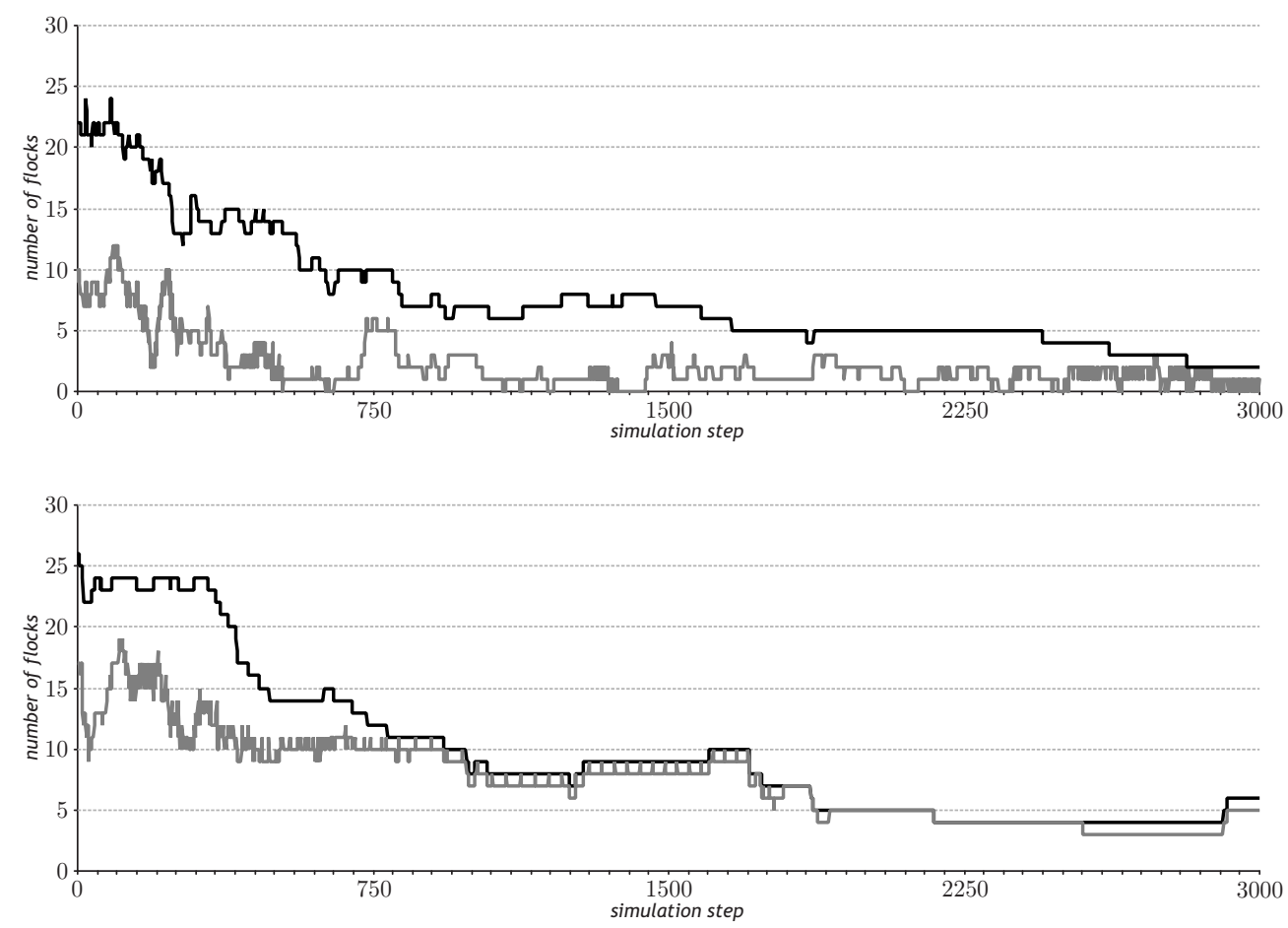

Fig. 10. Plot of the number of flocks (black line) and the contribution of leaderless flocks (grey line) for one of the eight experiments used to estimate the flocking ability of Reynolds's (1999) mathematical model (top chart) and our fuzzy model (bottom chart). Note that because in Reynolds's case the area of potential influence is larger, the number of flocks is lower to begin with and the values cannot be directly compared. However, since the number of flocks in both cases decreases through time we can conclude that the two models present flocking ability. Beside this, it can also be noticed that the fuzzy model produces mostly leaderless flocks whereas Reynolds's model mostly leader flocks.

\begin{tabular}{|c|c|c|c|c|c|c|c|c|}
\hline & \multicolumn{4}{|c|}{ Reynolds's model } & \multicolumn{4}{|c|}{ fuzzy model } \\
\hline & collisions & stragglers & flocks & $\begin{array}{l}\text { proportion } \\
\text { of leaderless } \\
\text { flocks (avg) }\end{array}$ & collisions & stragglers & flocks & $\begin{array}{l}\text { proportion } \\
\text { of leaderless } \\
\text { flocks (avg) }\end{array}$ \\
\hline 01 & 17 & 2 & 2 & $29 \pm 19$ & 0 & 1 & 6 & $83 \pm 14$ \\
\hline 02 & 25 & 2 & 5 & $30 \pm 17$ & 1 & 0 & 3 & $77 \pm 14$ \\
\hline 03 & 14 & 2 & 5 & $29 \pm 18$ & 14 & 0 & 3 & $90 \pm 12$ \\
\hline 04 & 14 & 2 & 5 & $27 \pm 18$ & 0 & 0 & 3 & $80 \pm 14$ \\
\hline 05 & 13 & 0 & 5 & $23 \pm 19$ & 0 & 1 & 5 & $82 \pm 17$ \\
\hline 06 & 10 & 1 & 4 & $31 \pm 18$ & 8 & 0 & 2 & $83 \pm 17$ \\
\hline 07 & 14 & 4 & 6 & $23 \pm 16$ & 0 & 0 & 3 & $77 \pm 15$ \\
\hline 08 & 18 & 7 & 5 & $26 \pm 16$ & 0 & 0 & 6 & $84 \pm 22$ \\
\hline avg & $15.63 \pm 4.5$ & $2.5 \pm 2.14$ & $4.63 \pm 1.19$ & $26.89 \pm 17.49$ & $2.88 \pm 5.28$ & $0.25 \pm 0.46$ & $3.88 \pm 1.55$ & $82.26 \pm 16.4$ \\
\hline
\end{tabular}

Table 1

A comparison of the number of collisions, number of stragglers and number of flocks after 3000 simulation steps and the average proportion of leaderless flocks in the eight experiments used for the estimation of flocking ability for Reynolds's (1999) mathematical model and our fuzzy model. Note that because in Reynolds's case the area of potential influence is larger, the number of flocks is lower to begin with and the values cannot be directly compared. 


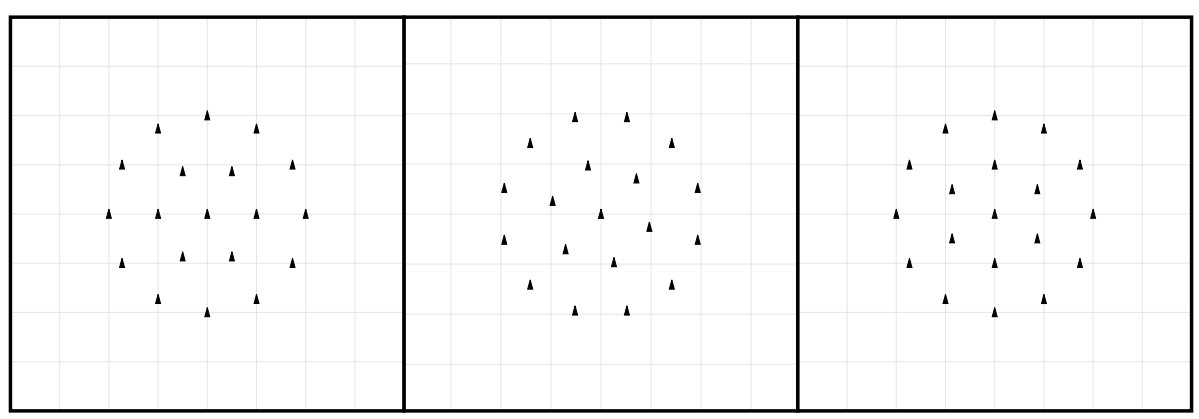

Fig. 11. Initial globular cluster distribution patterns used in the second set of experiments.

visual range used for the separation drive. The emerged flocks were regularly distributed and highly stable with no position shifting, which is, as already mentioned, the opposite of what can be seen in nature. In the rare cases when position shifting occured, it originated from the centre of the flock, subsided fast, and the flock stabilized immediately afterwards. The initial flock on average broke off to $1.3 \pm 0.61$ flocks and $0.44 \pm 1.09$ stragglers, with the first breakoff occurring at simulation step $500 \pm 395.85$. In our case, the initial flock on average broke off to $2.33 \pm 0.83$ flocks and no stragglers, with the first breakoff occurring in simulation step $3838.96 \pm 1680.98$. In all cases the animats from the initial flock formation started shifting positions and changing flock formation. The emerged front cluster formations were stable, but when animats due to position shifting and somewhat erratic and unsystematic behaviour reorganized into an extended cluster formation, they usually became disorganized and eventually broke off. With breakoffs flock formations from both major classes emerged. The resulting stable cluster flocks were of the front, globular and in few cases even extended cluster formation. The extended cluster formations were disorganized with frequent shifts of position. On the other hand, the resulting stable line flocks were of the ' $\mathrm{V}$ ', echelon and inverted ' $\mathrm{V}$ ' formation. In all cases these flocks were small, consisting of only two or three members and, with the only exception of the inverted ' $\mathrm{V}$ ' formation, they were all leader flocks. The above described behaviour is strikingly similar to natural flocks. If this bears up in future work it will make a very important point. In fact, all previous hypotheses about ' $\mathrm{V}$ ' formation flight assume a functional advantage: aerodynamics, visibility, communication (Heppner, 1974, 1997). The behaviour present in our experiments seems to suggest instead that even the ' $\mathrm{V}$ ' formation might be an emergent property.

In our third set of experiments we were interested if from a front line formation, where all animats have the same flight speed and flight direction, line formation flocks emerge. We used three initial distributions in which the animats were evenly spaced (approximately 2, 4 and 6 body lengths) and we changed the initial flight speed (10\%, $50 \%$ and $90 \%$ of maximum flight speed), that is nine initial states in all. Figure 13 presents a comparison of a series of time-equidistant frames from one of the experiments. In this set of experiments 


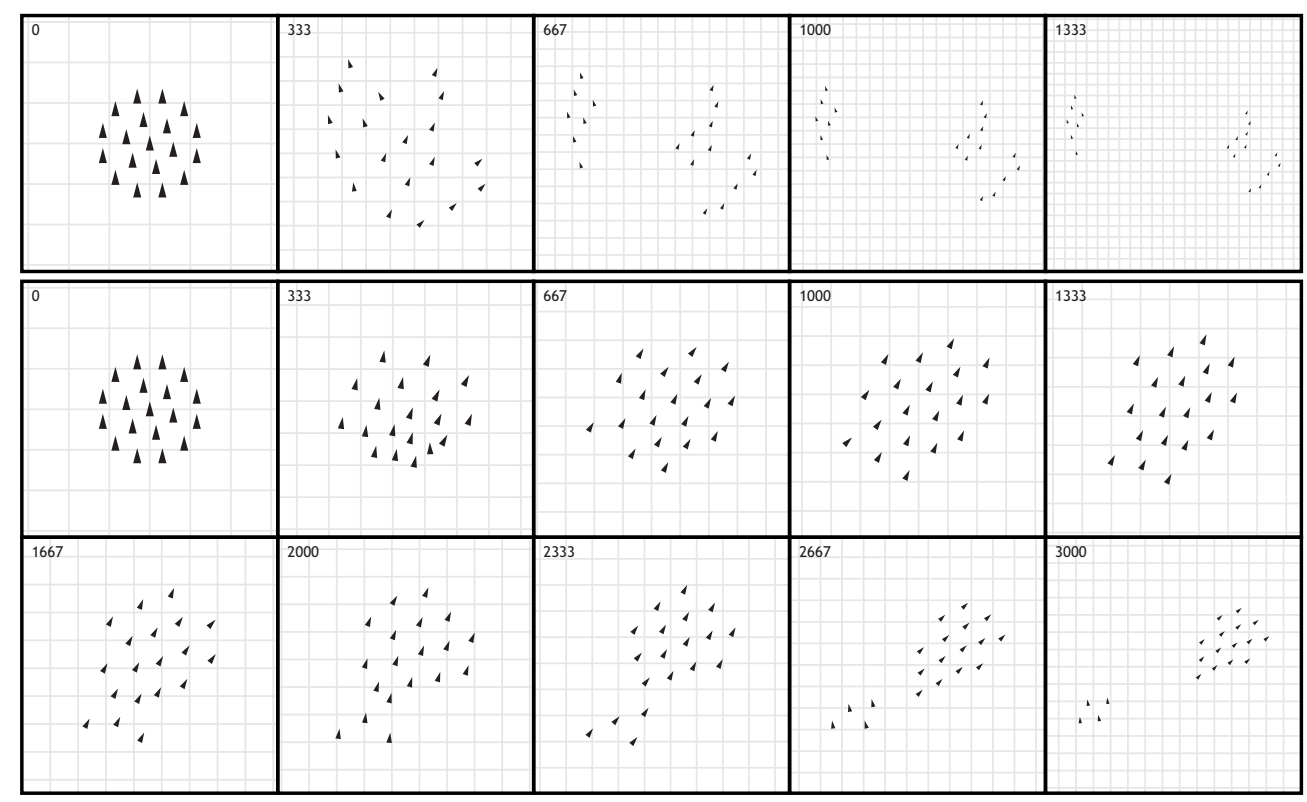

Fig. 12. A comparison of a series of time-equidistant frames from one of the 27 experiments with a globular cluster initial formation for Reynolds's mathematical model (top row) and our fuzzy model (middle and bottom row). The black triangles represent animats, with the apex indicating the flight direction. They were scaled for reasons of presentation clarity and their overlapping does not necessarily represent a collision. An animated version of this figure is available at the first author's web site.

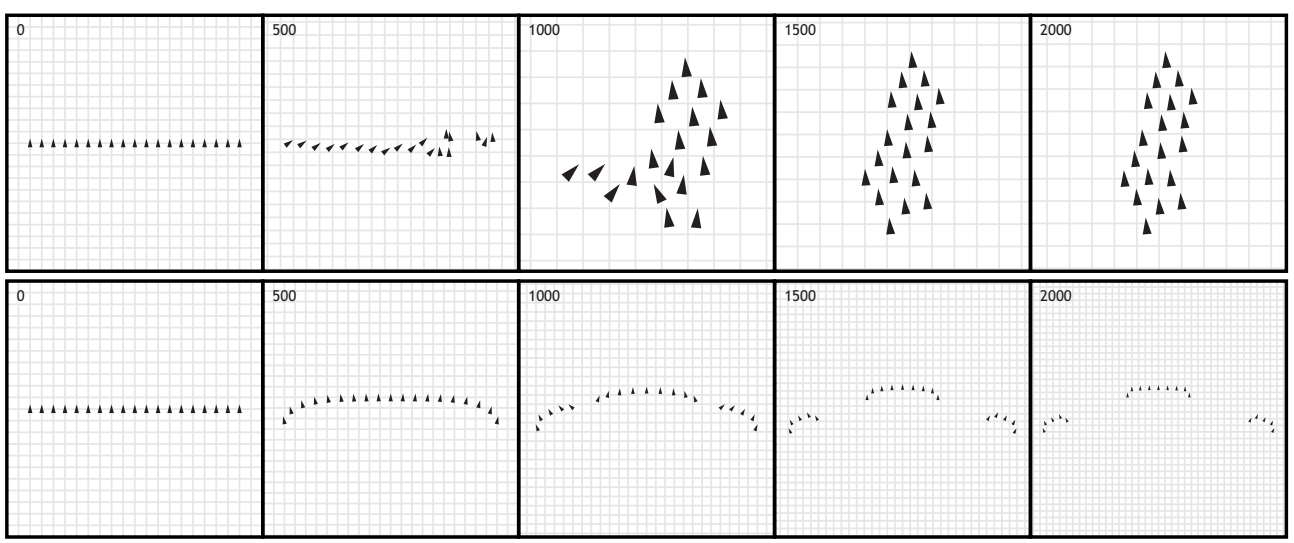

Fig. 13. A comparison of a series of time-equidistant frames from one of the nine experiments with an initial line formation for Reynolds's mathematical model (top row) and our fuzzy model (bottom row). The black triangles represent animats, with the apex indicating the flight direction. They were scaled for reasons of presentation clarity, which means that overlapping does not necessarily represent a collision. Notice that in the fuzzy model the ends of the flock trail away in a curve. This is a very natural behaviour. An animated version of this figure is available at the first author's web site. 
no roosting area was modelled. In Reynolds's case the initial flock broke off on average to $1.78 \pm 1.3$ flocks and $1 \pm 1.8$ stragglers and there were $0.33 \pm 0.5$ collisions. Most of the emerged flocks were of the extended cluster formation, regularly distributed and highly stable with no position shifting, but in some rare cases stable, regularly distributed column line formations emerged too. All of the emerged flocks were flying at maximum speed. In our case the initial flock broke off on average to $3.22 \pm 0.83$ flocks and $1.11 \pm 1.17$ stragglers with no collisions. All of the emerged flocks were of a formation that is similar to the ' $\mathrm{V}$ ' and front line formation type flying at approximatelly $71 \%$ of maximum speed.

\section{Conclusion}

In this paper we present a novel approach to the construction of artificial animals - animats, which is based on fuzzy logic. We believe that ethologists could use our method to gain additional insight into the behaviour of the animals of their interest. Knowledge about animal behaviour is usually available only in the form of the observer's linguistic descriptions and explanations of the perceived behaviour. Traditional approaches to modelling artificial animals are based on mathematical simulations. Their construction usually requires advanced mathematical skills and exact knowledge about the studied animal's behaviour. Our approach, on the other hand, is based on fuzzy logic, which allows us to model using uncertain knowledge and does not require the transition from linguistic descriptions to mathematical formulas. In section two we presented our approach through the study case of bird flocking. In section three, by comparing our model with Reynolds's (1987) mathematical model, we show that we can achieve comparable and in some aspects better and more natural behaviour even by using common sense knowledge about the behaviour of flocking birds. As our model currently uses unrealistic perception and action selection models our future work will focus on them as well as on extending our model to three dimensions. What is more, since we have recently attained the source code of the mathematical model used by Heppner and Grenander (1990), we plan a comparison with their model as our future work.

\section{Acknowledgements}

The work presented in this paper was done at the Computer Structures and Systems Laboratory, Faculty of Computer and Information Science, University of Ljubljana, Slovenia and is part of the $\mathrm{PhD}$ thesis that is being prepared by I. Lebar Bajec. 


\section{A Fuzzy Sets}

Fuzzy sets are a natural outgrowth and generalization of conventional (or crisp) sets. Recall that a crisp set in a universe of discourse (i.e. a collection of objects that represents allowable values for a variable) can be defined by stating all of its members or by specifying the precise properties required for membership. Take for example the set of real numbers 'from 3 to 5' denoted as C. In this case the universe of discourse is the set of real numbers $\mathbb{R}$ and $\mathbf{C}$ is a crisp set. We define this set by writing $\mathbf{C}=\{r \in \mathbb{R} \mid 3 \leq r \leq 5\}$. Equivalently, a crisp set can be described by specifying its membership function that maps from the universe of discourse to the set $\{0,1\}$. The membership function returns 1 for all objects from the universe of discourse that satisfy the required properties and 0 for those that do not. In our case the membership function returns 1 for all real numbers that satisfy the property 'from 3 to 5 ' and 0 for all other (Fig. A.1a). If we use $\mu_{\mathbf{C}}$ to denote the membership function of crisp set $\mathbf{C}$, then:

$$
\mu_{\mathbf{C}}(r)=\left\{\begin{array}{l}
1, \text { if } 3 \leq r \leq 5 \\
0, \text { otherwise }
\end{array}\right.
$$

Fuzzy sets, on the contrary, contain elements that satisfy imprecisely defined properties. Zadeh (1965) proposed describing them using a generalized membership function that maps from the universe of discourse to the entire unit interval $[0,1]$. This is the basic idea in fuzzy set theory; the membership function provides the degree to which an object from the universe of discourse satisfies the imprecisely defined properties (Bezdek and Pal, 1992). It provides the object's degree of membership. Take for example the 'set' of real numbers that are 'close to 4'. Because the property 'close to 4' is imprecise, the 'set' of real numbers that are 'close to 4' is a fuzzy set. Let us denote it by $\tilde{\mathbf{F}}$. The universe of discourse is the set of real numbers $\mathbb{R}$ again and the membership function $\mu_{\tilde{\mathbf{F}}}$ provides the degree of membership of a real number in the fuzzy set $\tilde{\mathbf{F}}$; the nearer the value of $\mu_{\tilde{\mathbf{F}}}(r)$ to unity the higher the degree of membership of $r$ in $\tilde{\mathbf{F}}$. This means that $\mu_{\tilde{\mathbf{F}}}(r)$ provides the measure of the degree of consistency between $r$ and our interpretation of 'close to 4'. However, since the property 'close to 4' is imprecise and its interpretation is subjective, there is not a unique membership function for $\tilde{\mathbf{F}}$. Rather, it is left to the modeller to decide what $\mu_{\tilde{\mathbf{F}}}$ should be like. In our particular case, with respect to the property 'close to 4', it seems plausible that:

(1) the degree of membership of number 4 is unity,

(2) the closer a number is to 4 , the closer its degree of membership is to 1 ,

(3) numbers equally far left and right of 4 have equal memberships. 

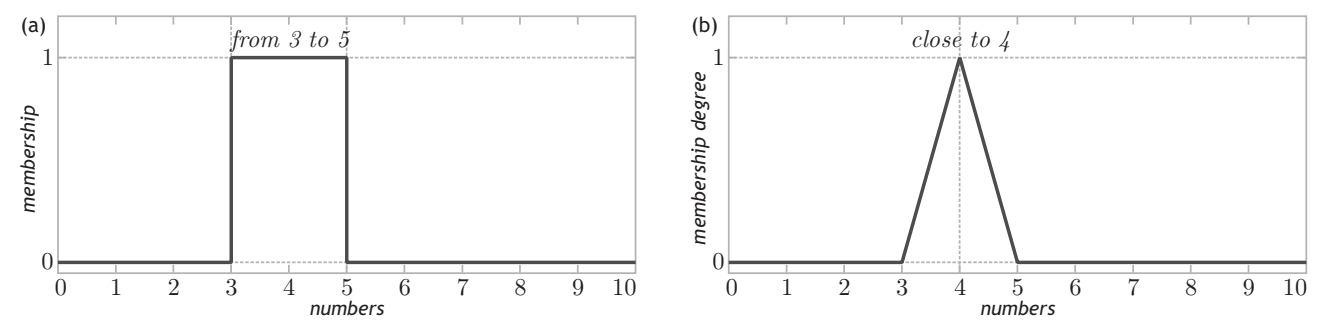

Fig. A.1. Membership functions of a crisp set of real numbers 'from 3 to 5' (a) and a fuzzy set of real numbers 'close to 4' (b).

Given these intuitive constraints, a useful representation of the fuzzy set of numbers 'close to 4' might be the fuzzy set $\tilde{\mathbf{F}}$ that is presented in Fig. A.1b and is described by the membership function

$$
\mu_{\tilde{\mathbf{F}}}(r)=\max (0,1-|r-4|) .
$$

\section{A.1 Set Theoretic Operations for Fuzzy Sets}

In order to manipulate fuzzy sets Zadeh (1965) generalized the classical set theoretic operations (i.e. intersection, union and complement) and introduced fuzzy intersection, fuzzy union, and fuzzy complement. Let fuzzy sets $\tilde{\mathbf{F}}_{1}$ and $\tilde{\mathbf{F}}_{2}$, defined on the universe of discourse $\mathbf{X}$, be described by their membership functions $\mu_{\tilde{\mathbf{F}}_{1}}$ and $\mu_{\tilde{\mathbf{F}}_{2}}$. The fuzzy set theoretic operations are then defined using the following operators:

- fuzzy intersection:

- minimum: $\mu_{\tilde{\mathbf{F}}_{1} \cap \tilde{\mathbf{F}}_{2}}(x)=\min \left(\mu_{\tilde{\mathbf{F}}_{1}}(x), \mu_{\tilde{\mathbf{F}}_{2}}(x)\right)$,

- algebraic product: $\mu_{\tilde{\mathbf{F}}_{1} \cap \tilde{\mathbf{F}}_{2}}(x)=\mu_{\tilde{\mathbf{F}}_{1}}(x) \cdot \mu_{\tilde{\mathbf{F}}_{2}}(x)$,

- fuzzy union:

- maximum: $\mu_{\tilde{\mathbf{F}}_{1} \cup \tilde{\mathbf{F}}_{2}}(x)=\max \left(\mu_{\tilde{\mathbf{F}}_{1}}(x), \mu_{\tilde{\mathbf{F}}_{2}}(x)\right)$,

- algebraic sum: $\mu_{\tilde{\mathbf{F}}_{1} \cup \tilde{\mathbf{F}}_{2}}(x)=\mu_{\tilde{\mathbf{F}}_{1}}(x)+\mu_{\tilde{\mathbf{F}}_{2}}(x)-\mu_{\tilde{\mathbf{F}}_{1}}(x) \cdot \mu_{\tilde{\mathbf{F}}_{2}}(x)$,

- fuzzy complement: $\mu_{\tilde{\mathbf{F}}_{1}}(x)=1-\mu_{\tilde{\mathbf{F}}_{1}}(x)$.

The minimum fuzzy intersection, maximum fuzzy union and fuzzy complement are also known under the names standard fuzzy intersection, standard fuzzy union and standard fuzzy complement. Later Klir and Yuan (1995) showed that by using a strong axiomatic basis many more operators can be defined. They even gave an axiomatic definition for the complement of a fuzzy set, but in engineering applications, most people prefer to use the standard fuzzy complement defined by Zadeh (1965). 


\section{B Fuzzy Logic}

The membership function of a crisp set maps real numbers to two values (0 or 1). Hence crisp sets correspond to two-valued logic. In logic we work with statements such as 'distance is from 3 to 5?'. This statement can be either true or false; there is no ambiguity in it. Let $d$ denote the value of distance and $\mathbf{C}$ denote the set of numbers 'from 3 to 5'. Then the question of the statement's truth becomes a question of membership 'is $d$ in $\mathbf{C}$ ?' and the answer is true if $\mu_{\mathbf{C}}(d)=1$ and false if $\mu_{\mathbf{C}}(d)=0$.

On the other hand, the value of $\mu_{\tilde{\mathbf{F}}}(r)$ provides the degree of membership of $r$ in fuzzy set $\tilde{\mathbf{F}}$. Therefore fuzzy sets correspond to continuously valued logic. Take for example the statement 'distance is close to 4?'. Because 'close to 4' is an imprecisely defined property, this statement is not crisp at all; we can not really tell if it is true or false. However, we can use a similar approach as in two-valued logic, denote the value of distance by $d$, but define 'close to 4 ' as a fuzzy set $\tilde{\mathbf{F}}$. Then the question of the statement's truth becomes again a question of membership 'is $d$ in $\tilde{\mathbf{F}}$ ?' but the value $\mu_{\tilde{\mathbf{F}}}(d)$ now answers the statement's degree of truth. The answer can be true $\left(\mu_{\tilde{\mathbf{F}}}(d)=1\right)$, false $\left(\mu_{\tilde{\mathbf{F}}}(d)=0\right)$ or anywhere in between $\left(0<\mu_{\tilde{\mathbf{F}}}(d)<1\right)$. Furthermore, in fuzzy logic the value of distance can be a fuzzy set $\tilde{\mathbf{D}}$ too. The statement's truth then becomes a question of similarity 'is $\tilde{\mathbf{D}}$ similar to $\tilde{\mathbf{F}}$ ?' and the answer is given by the highest degree of membership of objects that are common to both sets $\left(\sup _{r} \mu_{\tilde{\mathbf{D}} \cap \tilde{\mathbf{F}}}(r)\right)$.

Two-valued logic allows joining simple statements to form more complex ones. This is achieved through standard logical operators, namely 'and', 'or' and 'not'. An example of a complex statement could be '(distance is from 3 to 5 ) and (speed is $20 \mathrm{~m} / \mathrm{s}$ )'. Evaluating such statements involves computing the truths of the substatements and applying the logical operators. In two-valued logic the compound statement is true:

- when all of the substatements are true (logical operator 'and'),

- when at least one of the substatements is true (logical operator 'or'),

- when the substatement is false (logical operator 'not').

However, in fuzzy logic the constraint of the absolute truth or falsity of a statement is relaxed and this influences the interpretation of logical operators. Nevertheless, as fuzzy logic is a superset of standard two-valued logic, if we keep the degrees of truth at the extremes of 1 (completely true), and 0 (completely false), standard logical operators will hold. The most common interpretation is to use the operators that Zadeh (1965) used to define fuzzy intersection (logical operator 'and'), fuzzy union (logical operator 'or'), and fuzzy complement (logical operator 'not'). In other words this means that to 
resolve the truth of the statement ' $\mathcal{A}$ and $\mathcal{B}$ ', where $\mathcal{A}$ and $\mathcal{B}$ are statements and $T(\mathcal{A}), T(\mathcal{B})$ their corresponding degrees of truth, we evaluate the function

- minimum fuzzy intersection $T(\mathcal{A}$ and $\mathcal{B})=\min (T(\mathcal{A}), T(\mathcal{B}))$,

- algebraic product fuzzy intersection $T(\mathcal{A}$ and $\mathcal{B})=T(\mathcal{A}) \cdot T(\mathcal{B})$.

The degree of truth of ' $\mathcal{A}$ or $\mathcal{B}$ ' becomes equivalent

- maximum fuzzy union $T(\mathcal{A}$ or $\mathcal{B})=\max (T(\mathcal{A}), T(\mathcal{B}))$,

- algebraic sum fuzzy union $T(\mathcal{A}$ or $\mathcal{B})=T(\mathcal{A})+T(\mathcal{B})-T(\mathcal{A}) \cdot T(\mathcal{B})$.

Finally, the degree of truth of 'not $\mathcal{A}$ ' is computed as

- standard fuzzy complement $T($ not $\mathcal{A})=1-T(\mathcal{A})$.

Typically most fuzzy logic applications make use of these operators. But any combination of fuzzy union, fuzzy intersection and fuzzy complement operators can be used as far as we make sure that we either do not use DeMorgan's laws or that fuzzy union and fuzzy intersection are dual with respect to the chosen fuzzy complement (Klir and Yuan, 1995). This means that the different operators that are available to us in fuzzy set theory provide us with a plethora of richness, but also with some (tough) choices that have to be made (Mendel, 2001). In this paper we interpret the logical operator 'and' as product fuzzy intersection, the logical operator 'or' as maximum fuzzy union and 'not' as the standard fuzzy complement.

\section{B.1 The if-then Rule}

In logic we often use a special statement known as if-then rule, which assumes the form 'if $\mathcal{A}$ then $\mathcal{C}$ ', where $\mathcal{A}$ and $\mathcal{C}$ are statements. The if-part of the rule (i.e. statement $\mathcal{A}$ ) is called the antecedent or premise, while the then-part (i.e. statement $\mathcal{C}$ ) is called the consequent or conclusion. The antecedent can always be written as a set of statements connected using the logical operator 'and' (Mendel, 2001), which means that we can read the rule as a set of conditions that must be met for a certain consequence. However, this also means that antecedents of if-then rules that have the same conclusion can be joined using the logical operator 'or' and interpreted as a single if-then rule.

Interpreting an if-then rule involves evaluating the truth of the antecedent and applying that result to the consequent (known as implication). In the case when the antecedent has multiple parts, their degrees of truth are calculated simultaneously and the truth of the antecedent is resolved by applying the logical operators. In two-valued logic the interpretation of the if-then rule is simple. Whenever the premise is true the conclusion is true too. However, if 

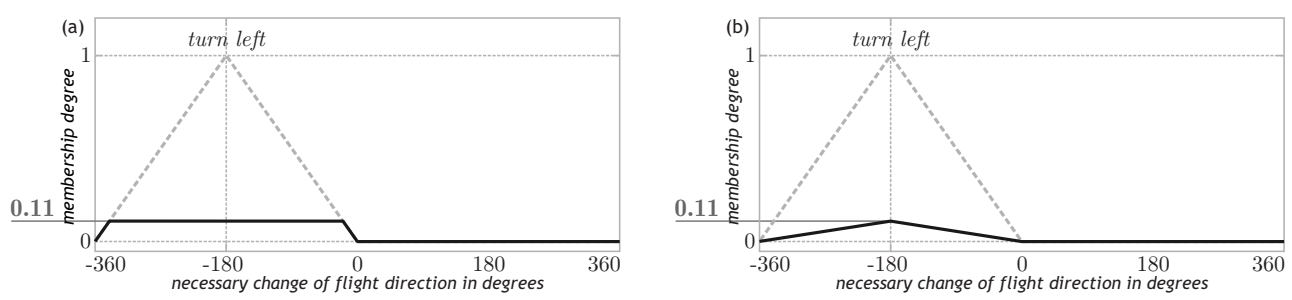

Fig. B.1. Graphical representation of the minimum (a) and product (b) fuzzy implication. The dashed grey line represents the original output fuzzy set while the black solid line represents the modified output fuzzy set.

the premise is false we can not say anything about the conclusion. Since in fuzzy logic the truth of the antecedent is a matter of degree, the interpretation of the if-then rule is less restricted. Whenever the antecedent is true to some degree, the consequent is also true to that same degree.

However, because in fuzzy logic the consequent specifies a fuzzy set to be assigned to the output, implication modifies this set to the degree specified by the antecedent. The most common ways to modify the output fuzzy set are chopping it off (i.e. minimum fuzzy implication) or squashing it (i.e. product fuzzy implication). Let $T(\mathcal{A})$ denote the antecedent's degree of truth and $\tilde{\mathbf{F}}$ be the output fuzzy set defined on the real numbers domain. Let $\mu_{\tilde{\mathbf{F}}}$ denote the membership function of the output fuzzy set and $\mu_{\tilde{\mathbf{F}}^{\prime}}$ the membership function of the modified output fuzzy set. Then minimum fuzzy implication is computed as

$$
\mu_{\tilde{\mathbf{F}}^{\prime}}(r)=\min \left(T(\mathcal{A}), \mu_{\tilde{\mathbf{F}}}(r)\right)
$$

and product fuzzy implication as

$$
\mu_{\tilde{\mathbf{F}}^{\prime}}(r)=T(\mathcal{A}) \cdot \mu_{\tilde{\mathbf{F}}}(r) .
$$

Take for example the if-then rule whose consequent is 'direction is turn left' and let the degree of truth of its antecedent be $T(\mathcal{A})=0.11$. The minimum fuzzy implication's effect of chopping off the output fuzzy set can be seen in Fig. B.1a while the squashing effect of the product fuzzy implication can be seen in Fig. B.1b.

\section{Computing the Animat's Action}

Let there be an animat that perceives only two neighbours, one of which is $80 \%$ of the visual range away and has an angular offset of $-30^{\circ}\left(\mathrm{N}_{0}\right)$ and the other is $60 \%$ of the visual range away and has an angular offset of $-110^{\circ}\left(\mathrm{N}_{1}\right)$ 


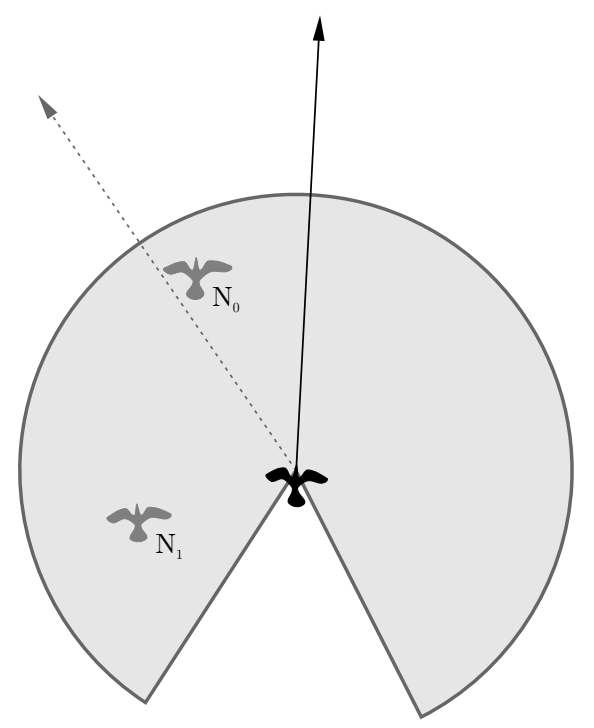

Fig. C.1. The animat perceives two neighbours, one of which is $80 \%$ of the visual range away with an angular offset of $-30^{\circ}\left(\mathrm{N}_{0}\right)$, and the other is $60 \%$ of the visual range away with an angular offset of $-110^{\circ}\left(\mathrm{N}_{1}\right)$. The black arrow represents the animat's current flight direction. The dashed grey arrow represents the flight direction change that the animat obtained by applying fuzzy logic to the rule base of the attraction drive.

(see Fig. C.1). For reasons of simplicity we shall assume that all are flying in the same direction and with the same flight speed.

By applying fuzzy logic on the attraction, repulsion and polarisation drive if-then rule bases the animat computes three independent actions (i.e. flight direction and flight speed changes). Together, these actions will satisfy its drives to stay close to the perceived neighbours, keep away from colliding with them and fly in approximately the same direction and flight speed. The animat combines, prioritizes and arbitrates these actions and computes the flight direction and flight speed change to be taken in the following time step.

Even though all rule bases can be evaluated simultaneously, let us start with the attraction drive. For each of the perceived neighbours the rule base is evaluated independently (i.e. as if the animat perceived only one neighbour) and all outputs are later combined. Now, recall that the action that satisfies the attraction drive depends only on the perceived neighbour's distance and position. Because all of the perceived information is precise, for neighbour $\mathrm{N}_{0}$, distance is the crisp value $80 \%$ of the visual range and position is the crisp value $-30^{\circ}$.

First let us compute the necessary change in flight direction (i.e. evaluate rules a1,a2,a6 and a8). It is easy to notice that rules a1 and a2 have the same consequent (i.e. 'distance is keep direction'). Because of this their antecedents can be joined, using the logical operator 'or', and interpreted as a single 
rule. To assess the degree of truth of the compound antecedent we must first compute the degrees of truth of the individual antecedents and then apply the fuzzy logic operator 'or'.

Let $\mathcal{A}_{1}$ denote the antecedent of rule a1 (i.e. 'distance is close enough') and $\mathcal{A}_{2}$ the antecedent of rule a2 (i.e. 'distance is too far'). Recall that as distance is a crisp value, the degrees of truth of $\mathcal{A}_{1}$ and $\mathcal{A}_{2}$ are given by the degree of membership of distance in the corresponding fuzzy sets. Therefore, if we use $d$ to denote the value of distance, $\tilde{\mathbf{C}}$ to denote the fuzzy set 'close enough' and $\tilde{\mathbf{F}}$ the fuzzy set 'too far', then

$$
\begin{aligned}
& T\left(\mathcal{A}_{1}\right)=\mu_{\tilde{\mathbf{C}}}(d)=\mu_{\tilde{\mathbf{C}}}(80)=0.33 \\
& T\left(\mathcal{A}_{2}\right)=\mu_{\tilde{\mathbf{F}}}(d)=\mu_{\tilde{\mathbf{F}}}(80)=0.67
\end{aligned}
$$

Since we interpret the logical operator 'or' as maximum fuzzy union, the degree of truth of the compound antecedent is

$$
T\left(\mathcal{A}_{1} \text { or } \mathcal{A}_{2}\right)=\max \left(T\left(\mathcal{A}_{1}\right), T\left(\mathcal{A}_{2}\right)\right)=\max (0.33,0.67)=0.67 .
$$

Recall that the degree of truth of the antecedent implies the degree of truth of the conclusion, and that fuzzy implication modifies the output fuzzy set. In this paper we use product fuzzy implication, which modifies the output fuzzy set by squashing it. Let $\mu_{\tilde{\mathbf{K}}}$ denote the fuzzy set 'keep direction'. Then the membership function of the modified output fuzzy set that results from rules a1 and a2 is given by

$$
\mu_{\tilde{\mathbf{K}}^{\prime}}(r)=T\left(\mathcal{A}_{1} \text { or } \mathcal{A}_{2}\right) \cdot \mu_{\tilde{\mathbf{K}}}(r)=0.67 \cdot \mu_{\tilde{\mathbf{K}}}(r) .
$$

A graphical representation of the above described evaluation process is presented in Fig. C.2. The left half shows the evaluation of the antecedents' degrees of truth, while the right shows the modification of the output fuzzy set.

Rules a6 and a8 have different consequents, which means that, even though they can be evaluated simultaneously, they can not be treated as a single rule. However, we notice that their antecedents have multiple parts. In both cases the antecedent is composed of two conditions joined by the logical operator 'and'. This means that to evaluate the degree of truth of the antecedent we must first compute the degrees of truth of the individual conditions and then apply the fuzzy logic operator 'and'. A graphical representation of the evaluation process is presented in Fig. C.3.

For rule a6 let $\mathcal{A}_{61}$ denote condition 'distance is too far' and $\mathcal{A}_{62}$ condition 'position is left or behind'. Then, if we use $d$ to denote the value of distance, 


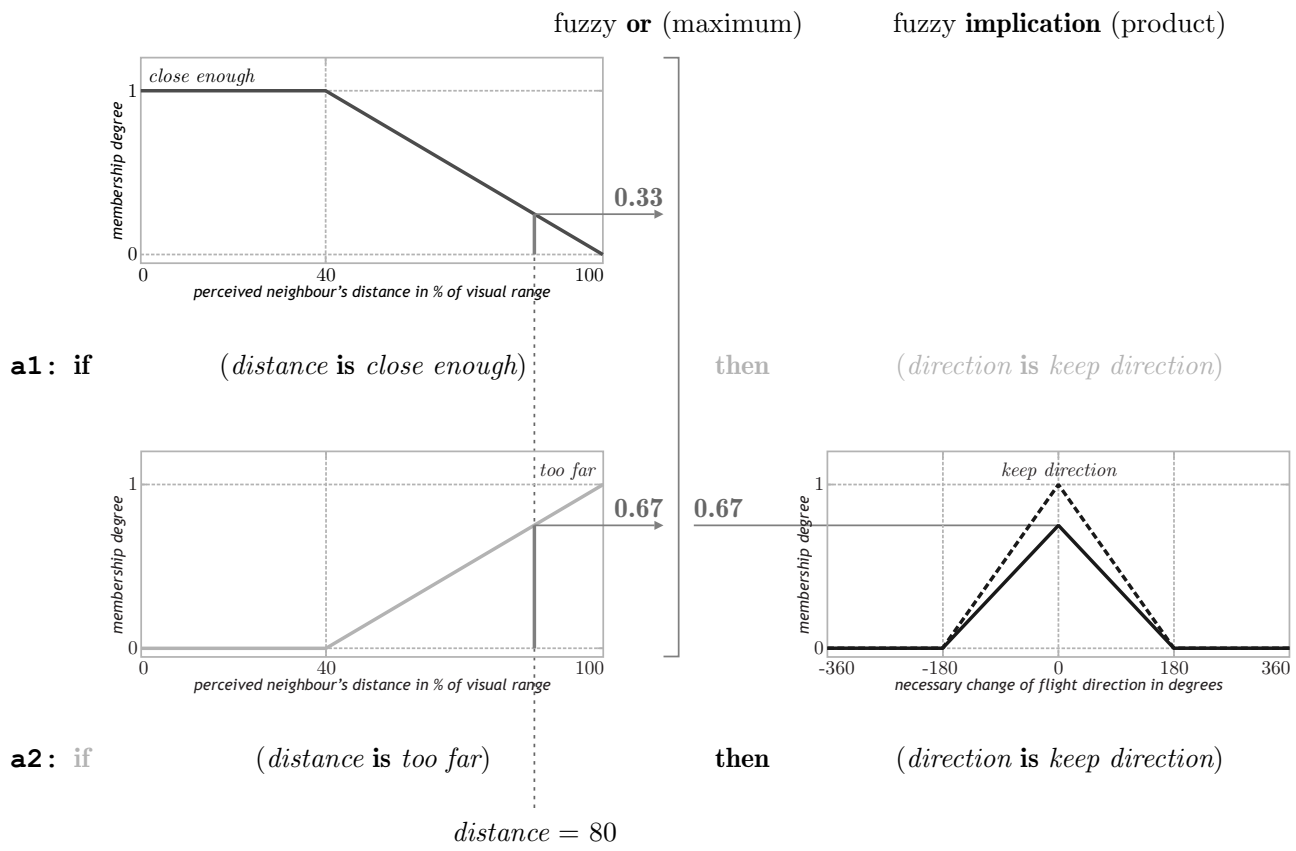

Fig. C.2. Graphical representation of the evaluation of rules a1 and a2 for the case when the perceived neighbour is $80 \%$ of the visual range away with an angular offset of $-30^{\circ}$. The left half shows the evaluation of the degrees of truth of the antecedents and the right part the modification of the output fuzzy set.

$p$ to denote the value of position, $\tilde{\mathbf{F}}$ the fuzzy set 'too far' and $\tilde{\mathbf{L}}$ the fuzzy set 'left or behind', we can write

$$
\begin{aligned}
& T\left(\mathcal{A}_{61}\right)=\mu_{\tilde{\mathbf{F}}}(d)=\mu_{\tilde{\mathbf{F}}}(80)=0.67, \\
& T\left(\mathcal{A}_{62}\right)=\mu_{\tilde{\mathbf{L}}}(p)=\mu_{\tilde{\mathbf{L}}}(-30)=0.17,
\end{aligned}
$$

and, because we use product fuzzy intersection to interpret the logical operator 'and', the degree of truth of the antecedent of rule a6 is

$$
T\left(\mathcal{A}_{61} \text { and } \mathcal{A}_{62}\right)=T\left(\mathcal{A}_{61}\right) \cdot T\left(\mathcal{A}_{62}\right)=0.67 \cdot 0.17=0.11
$$

This means that, if we use $\tilde{\mathbf{T}}_{\mathrm{L}}$ to denote the fuzzy set 'turn left', the membership function of the modified output fuzzy set that results from rule a6 is

$$
\mu_{\tilde{\mathbf{T}}_{\mathrm{L}}^{\prime}}(r)=T\left(\mathcal{A}_{61} \text { and } \mathcal{A}_{62}\right) \cdot \mu_{\tilde{\mathbf{T}}_{\mathrm{L}}}(r)=0.11 \cdot \mu_{\tilde{\mathbf{T}}_{\mathbf{L}}}(r) .
$$

Similarly for rule a8 we denote condition 'distance is too far' as $\mathcal{A}_{81}$ and condition 'position is right or behind' as $\mathcal{A}_{82}$. Then, if we denote the fuzzy set 


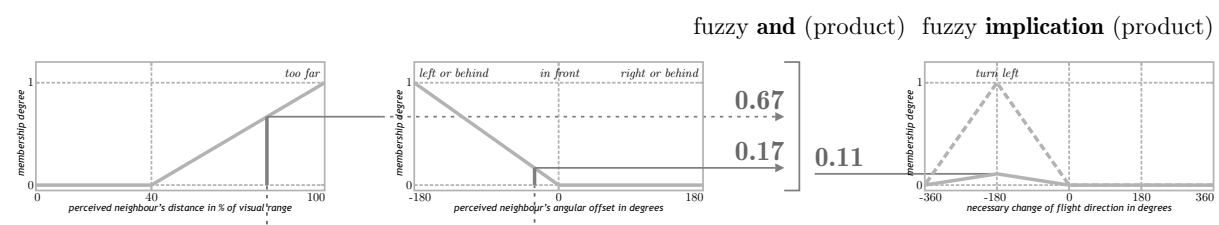

a6: if

(distance is too far) and (position is left or behind)

then (direction is turn left)
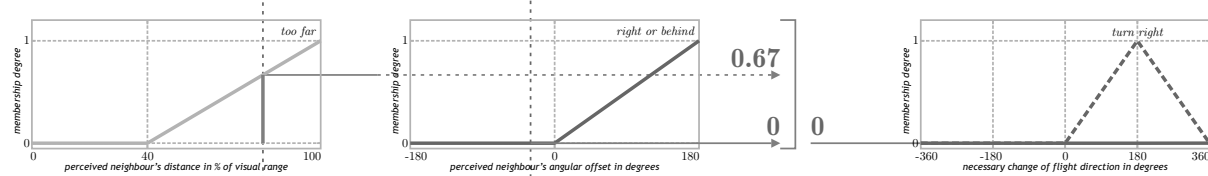

a8: if

(distance is too far) and (position is right or behind)

then (direction is turn right)

$$
\text { distance }=80 \quad \text { position }=-30
$$

Fig. C.3. Graphical representation of the evaluation of rules a 6 and a8 for the case when the perceived neighbour is $80 \%$ of the visual range away with an angular offset of $-30^{\circ}$. The left half shows the evaluation of the degrees of truth of the antecedents and the right part the modification of the output fuzzy sets.

'right or behind' as $\tilde{\mathbf{R}}$, we can write

$$
\begin{aligned}
& T\left(\mathcal{A}_{81}\right)=\mu_{\tilde{\mathbf{F}}}(d)=\mu_{\tilde{\mathbf{F}}}(80)=0.67, \\
& T\left(\mathcal{A}_{82}\right)=\mu_{\tilde{\mathbf{R}}}(p)=\mu_{\tilde{\mathbf{R}}}(-30)=0,
\end{aligned}
$$

and the degree of truth of the antecedent of rule a8 is

$$
T\left(\mathcal{A}_{81} \text { and } \mathcal{A}_{82}\right)=T\left(\mathcal{A}_{81}\right) \cdot T\left(\mathcal{A}_{82}\right)=0.67 \cdot 0=0,
$$

which means that the antecedent is false.

For neighbour $\mathrm{N}_{1}$ the membership function of the modified output fuzzy set that results from rules a1 and a2 is given by

$$
\begin{aligned}
\mu_{\tilde{\mathbf{K}}^{\prime}}(r) & =T\left(\mathcal{A}_{1} \text { or } \mathcal{A}_{2}\right) \cdot \mu_{\tilde{\mathbf{K}}}(r)=\max \left(\mu_{\tilde{\mathbf{C}}}(60), \mu_{\tilde{\mathbf{F}}}(60)\right) \cdot \mu_{\tilde{\mathbf{K}}}(r)= \\
& =\max (0.67,0.33) \cdot \mu_{\tilde{\mathbf{K}}}(r)=0.67 \cdot \mu_{\tilde{\mathbf{K}}}(r)
\end{aligned}
$$

the modified output fuzzy set that results from rule a6 is

$$
\begin{aligned}
\mu_{\tilde{\mathbf{T}}_{\mathrm{L}}^{\prime}}(r) & =T\left(\mathcal{A}_{61} \text { and } \mathcal{A}_{62}\right) \cdot \mu_{\tilde{\mathbf{T}}_{\mathrm{L}}}(r)= \\
& =\left(\mu_{\tilde{\mathbf{F}}}(60) \cdot \mu_{\tilde{\mathbf{L}}}(-110)\right) \cdot \mu_{\tilde{\mathbf{T}}_{\mathrm{L}}}(r)= \\
& =(0.33 \cdot 0.61) \cdot \mu_{\tilde{\mathbf{T}}_{\mathbf{L}}}(r)=0.2 \cdot \mu_{\tilde{\mathbf{T}}_{\mathrm{L}}}(r),
\end{aligned}
$$

whereas the antecedent of rule a8 is again false. 
neighbour $\mathrm{N}_{0}$
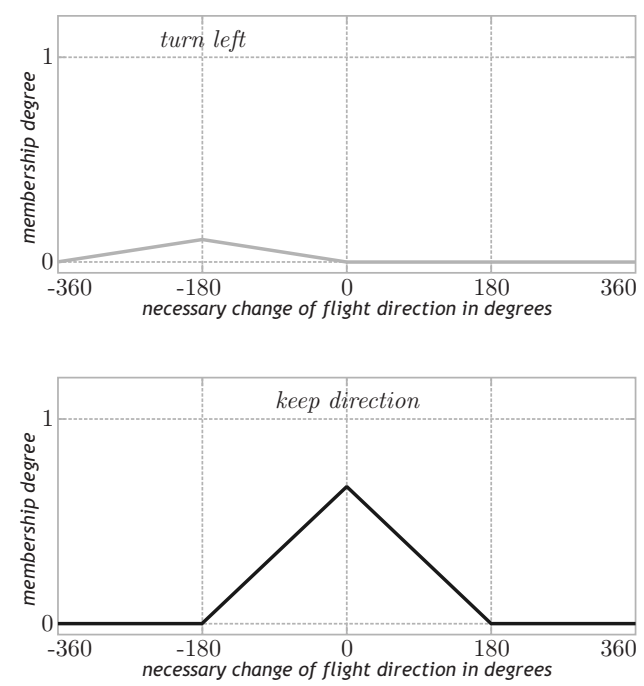

neighbour $\mathrm{N}_{0}$
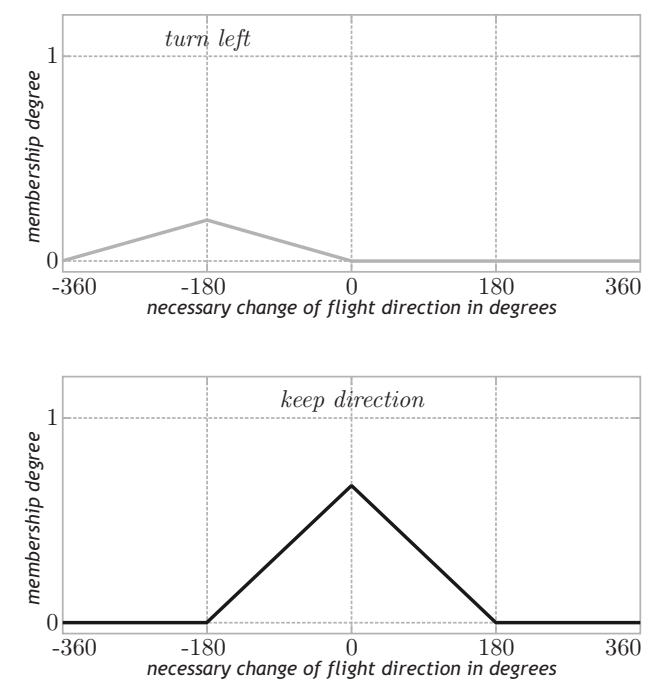

combined

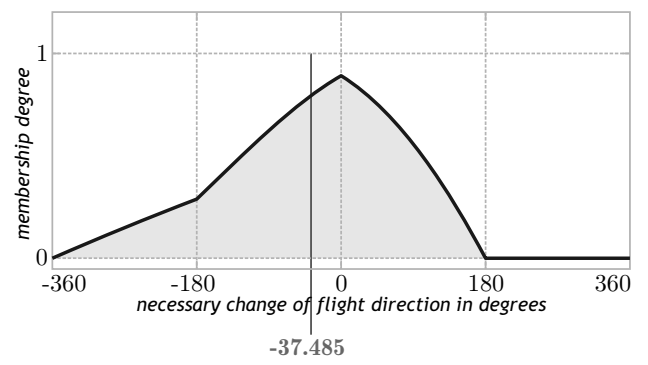

Fig. C.4. The modified output fuzzy sets that result from active rules. They were obtained by evaluating the attraction drive rule base for the case when the only two perceived neighbours are $80 \%$ of the visual range away with an angular offset of $-30^{\circ}\left(\mathrm{N}_{0}\right)$, and $60 \%$ of the visual range away with an angular offset of $-110^{\circ}$ $\left(\mathrm{N}_{1}\right)$. The combined fuzzy set is the algebraic sum fuzzy union of the modified output fuzzy sets. The defuzzified value, obtained by using the centroid method, is $-37.485^{\circ}$.

Rules whose antecedents are true to a non-zero degree are active rules. The modified output fuzzy set that results from the consequent of an active rule represents the flight direction change according to that rule, expressed in the form of a fuzzy set. But because in fuzzy logic more than one rule can be active at a time and because we evaluate the rules for each perceived neighbour individually, all of the modified output fuzzy sets have to be combined into a single fuzzy set (Fig. C.4). This is done by computing the fuzzy union of the modified output fuzzy sets and, in this paper, we use the algebraic sum fuzzy union (see Appendix A). The combined fuzzy set that was obtained by evaluating the rule base for the two perceived neighbours is presented in Fig. C.4.

However, even though the combined fuzzy set represents the flight direction change that will help satisfy the attraction drive it is still a fuzzy set. Therefore, 
in the final step, it has to be converted into a single (crisp) value that, in some sense, is the best representative of the fuzzy set. This conversion is called defuzzification.

A number of defuzzification methods leading to distinct results were proposed in literature, but the most commonly used, and also the one used in this paper, is called the centroid method. In this method, which is sometimes called the centre of gravity or centre of area method, the defuzzified value is defined as the crisp value, for which the area under the graph of the membership function of the combined fuzzy set is divided into two equal subareas. Let $\tilde{\mathbf{D}}$ denote the combined fuzzy set, then the centroid defuzzified value is calculated using the formula

$$
\operatorname{cog}(\tilde{\mathbf{D}})=\frac{\int r \cdot \mu_{\tilde{\mathbf{D}}}(r) \mathrm{d} r}{\int \mu_{\tilde{\mathbf{D}}}(r) \mathrm{d} r}
$$

If we return to our example, the defuzzified value resulting from the combined fuzzy set presented in Fig. C.4 is $-37.485^{\circ}$. This means that in order to satisfy the attraction drive the animat should turn to the left by $37.485^{\circ}$. By computing the necessary change in flight speed we find out that, in order to satisfy the attraction drive, the animat should also increase its flight speed by $17.3489 \%$ of max speed. The repulsion drive will be satisfied with a turn to the right by $11.078^{\circ}$ and a speed increase of $4.3825 \%$ of max speed. However, since the animat and the perceived neighbours have the same flight direction and flight speed, polarization drive does not require any changes.

By combining, prioritizing, and arbitrating between these actions, action selection selects the actual change in flight direction and flight speed to be taken. Since we do not model the musculoskeletal structure of the bird, the animat is based on a point mass approximation. The same approach, named a point mass vehicle model, was used by Reynolds (1999). The animat's physics is thus based on forward Euler integration. At each simulation step for each drive a vector is calculated that represents the force needed to accomplish the required action. These forces are combined by using a weighted sum and the resulting force (limited by the animat's available force) is applied to the animat's point mass. This produces an acceleration equal to the force divided by the animat's mass. The acceleration is then added to the animat's current velocity vector and truncated by the maximum achievable speed. Finally the animat's new position is computed by adding the new velocity vector to the animat's current position. 


\section{References}

Bezdek, J.C., Pal, S.K., 1992. Fuzzy Models For Pattern Recognition: Methods That Search for Structures in Data. IEEE Press, New York.

Heppner, F., 1974. Avian flight formations. Bird-Banding 45 (2), 160-169.

Heppner, F., Convissar, J.L., Moonan, D.E., Anderson, J.G.T., 1985. Visual angle and formation flight in Canada Geese (Branta canadensis). The AUK 102 (1), 195-198.

Heppner, F., 1987. A stochastic nonlinear model for coordinated bird flocks. American Ornithological Union Meeting (abstract), San Francisco.

Heppner, F., Grenander, U., 1990. A stochastic nonlinear model for coordinated bird flocks, in: Krasner, S., (Ed.), The Ubiquity of Chaos, AAAS, Washington, pp. 233-238.

Heppner, F., 1997. Three-dimensional structure and dynamics of bird flocks, in: Parrish, J.K., Hamner, W.M., (Eds.), Animal Groups in Three Dimensions, Cambridge University Press, United Kingdom, pp. 68-89.

Jaffe, J.S., 1997. Methods for three-dimensional sensing of animals, in: Parrish, J.K., Hamner, W.M., (Eds.), Animal Groups in Three Dimensions, Cambridge University Press, United Kingdom, pp. 17-35.

Klir, G.J., Yuan, B., 1995. Fuzzy Sets and Fuzzy Logic: Theory and Applications, Prentice-Hall, New Jersey.

Lebar Bajec, I., 2002. Computer Model of Bird Flocking, MSc thesis, Faculty of Computer and Information Science, Ljubljana, Slovenia.

Lebar Bajec, I., Zimic, N., Mraz, M., 2003. Fuzzifying the thoughts of animats, in: Bilgic, T., De Baets, B., Kaynak, O., (Eds.), Proc. of IFSA 2003, (LNAI 2715), Springer-Verlag, Heidelberg, Germany, pp. 195-202.

Lebar Bajec, I., Zimic, N., Mraz, M., 2003. Boids with a fuzzy way of thinking, in: Leung, H., (Ed.), Proc. of ASC 2003, ACTA Press, Anaheim; Calgary; Zurich, pp. 58-62.

Lee, S., Lee, K.H., Lee, D., 2004. Ranking the sequences of fuzzy values. Information Sciences 160, 41-52., doi:10.1016/j.ins.2003.07.011.

Mendel, J.M., 2001. Uncertain Rule-Based Fuzzy Logic Systems: Introduction and New Directions, Prentice-Hall, Inc., New York.

Mraz, M., Zimic, N., Virant, J., 1999. Intelligent bush fire spread prediction using fuzzy cellular automata. Journal of Intelligent and Fuzzy Systems 7 (2), 203-207.

Mraz, M., Lebar Bajec, I., Zimic, N., 2004. Fuzzy logic used for modelling and simulation of massive processes: A useful tool for nanotechnologies?, in: Hamza, M.H., (Ed.), Proc. of NNCI 2004, ACTA Press, Anaheim; Calgary; Zurich, pp. 274-278.

Okubo, A., 1980. Diffusion and Ecological Problems: Mathematical Models, (Biomathematics 10) Springer-Verlag, Berlin; Heidelberg; New York.

Okubo, A., 1986. Dynamical aspects of animal grouping: swarms, schools, flocks and herds. Advances in Biophysics 22, 1-94., doi:10.1016/0065227X(86)90003-1. 
Parrish, J.K., Hamner, W.M., Prewitt, C.T., 1997. Introduction - From individuals to aggregations: Unifying properties, global framework and the holy grails of congregation, in: Parrish, J.K., Hamner, W.M., (Eds.), Animal Groups in Three Dimensions, Cambridge University Press, United Kingdom, pp. 1-13.

Pomeroy, H., Heppner, F.H., 1992. Structure of turning in a flock of rock doves. The AUK 109 (2), 256-267.

Rackham, H., 1933. Pliny's Natural History, Book X:XXXV, Harvard University Press, Cambridge, MA.

Reynolds, C.W., 1987. Flocks, herds, and schools: A distributed behavioral model. Computer Graphics (SIGGRAPH 1987 Conf. Proc.) 21 (4), 25-34.

Reynolds, C.W., 1993a. An evolved, vision-based behavioral model of coordinated group motion, in: Meyer, J.A., Roitblat, H.L., Wilson, S.W., (Eds.), From Animals to Animats 2: Proc. of SAB92, MIT Press, Cambridge, MA, pp. 384-392.

Reynolds, C.W., 1993b. An evolved, vision-based model of obstacle avoidance behavior, in: Langton, C., (Ed.), Artificial Life III, Addison-Wesley, Redwood City, CA, pp. 327-346.

Reynolds, C.W., 1994. Evolution of obstacle avoidance behavior: using noise to promote robust solutions, in: Kinnear, Jr., K.E., (Ed.), Advances in Genetic Programming, MIT Press, Cambridge, MA, pp. 221-242.

Reynolds, C.W., 1999. Steering behaviors for autonomous characters, in: Proc. of GDC 1999, Miller Freeman Game Group, San Francisco, CA, pp. 763782.

Reynolds, C.W., 2001. Interaction with groups of autonomous characters, in: Proc. of GDC 2001, CMP Game Media Group, San Francisco, CA, pp. 449-460.

Spector, L., Klein, J., 2002. Evolutionary dynamics discovered via visualization in the BRAVE simulation environment, in: Bilotta, E. et al. (Eds.), Workshop Proc. of ALife VIII, UNSW Press, Sydney, pp. 163-170.

Spector, L., Klein, J., Perry, C., Feinstein, M., 2003. Emergence of collective behavior in evolving populations of flying agents, in: Cantú-Paz, E. et al. (Eds.), Proc. of GECCO 2003, (LNCS 2723) Springer-Verlag, Berlin; Heidelberg; New York, pp. 61-73.

Turchin, P., 1997. Quantitative Analysis of Movement, Sinauer Associates, Sunderland, MA.

Vakalis, D., Sarimveis, H., Kiranoudis, C., Alexandridis, A., Bafas, G., 2004a. A GIS based operational system for wildland fire crisis management I: Mathematical modelling and simulation. Applied Mathematical Modelling 28 (4), 389-410., doi:10.1016/j.apm.2003.10.005.

Vakalis, D., Sarimveis, H., Kiranoudis, C., Alexandridis, A., Bafas, G., 2004b. A GIS based operational system for wildland fire crisis management II: System architecture and case studies. Applied Mathematical Modelling 28 (4), 411-425., doi:10.1016/j.apm.2003.10.006.

Wilson, S.W., 1985. Knowledge growth in an artificial animal, in: Grefenstette, 
J.J., (Ed.), Proc. of ICGA 1985, Lawrence Erlabum Assoc, Hilsdale, NY, pp. 16-23.

Zadeh, L.A., 1965. Fuzzy sets. Information and Control 8 (3), 338-353. 